\title{
Modeling the cross power spectrum of the Sunyaev-Zel'dovich and X-ray surveys
}

\author{
G. Hurier, N. Aghanim, and M. Douspis
}

\begin{abstract}
Institut d'Astrophysique Spatiale, CNRS (UMR 8617) and Université Paris-Sud 11, Bâtiment 121, 91405 Orsay, France
e-mail: ghurier@ias.u-psud.fr
\end{abstract}

Received 5 March 2014 / Accepted 25 May 2014

\begin{abstract}
The thermal Sunyaev-Zel'dovich (tSZ) effect and X-ray emission from galaxy clusters have been used extensively to constrain cosmological parameters. These constraints are highly sensitive to the relations between cluster masses and observables (tSZ and X-ray fluxes). The cross-correlation of tSZ and X-ray data is thus a powerful tool, in addition of tSZ and X-ray based analysis, for testing our modeling of both tSZ and X-ray emission from galaxy clusters. We chose to explore this cross-correlation because both emissions trace the hot gas in galaxy clusters and thus constitute one of the easiest correlations that can be studied. We present a complete modeling of the cross-correlation between tSZ effect and X-ray emission from galaxy clusters and focus on the dependencies with cluster scaling laws and cosmological parameters. We show that current knowledge of cosmological parameters and scaling-law parameters leads to uncertainties of $48 \%$ on the overall normalization of the tSZ-X cross-correlation power spectrum. We present the expected signal-to-noise ratio for the tSZ-X cross-correlation angular power spectrum and consider the sensitivity of actual tSZ and X-ray surveys from Planck-like data and ROSAT. We demonstrate that this signal-to-noise can reach 31.5 in a realistic situation, leading to a constraint on the amplitude of tSZ-X cross-correlation up to $3.2 \%$, which is ten times better than actual modeling limitations. Consequently, using it in addition to other probes of cosmological parameters and scaling relations, we show that the tSZ-X is a powerful probe that constrains the cosmological parameters of scaling relations.
\end{abstract}

Key words. galaxies: clusters: general - X-rays: galaxies: clusters - submillimeter: general - large-scale structure of Universe galaxies: clusters: intracluster medium

\section{Introduction}

Galaxy clusters are the largest virialized structures. They can be observed through X-ray, via the bremsstrahlung emission produced by the ionized intracluster medium (see, e.g., Bohringer et al. 2000; Ebeling et al. 2000, 2001). This hot intracluster medium also produces a distortion of the cosmic microwave background (CMB) black-body emission via the thermal Sunyaev-Zel'dovich (tSZ) effect (Sunyaev \& Zeldovich 1969, 1972). This effect was observed toward a large number of clusters by Planck (Planck Collaboration VIII 2011; Planck Collaboration XXIX 2014), ACT (Marriage et al. 2011), and SPT (Reichardt et al. 2013).

The number of galaxy clusters is extremely sensitive to cosmological parameters, especially to the normalization of the matter power spectrum, $\sigma_{8}$, and to the matter density, $\Omega_{\mathrm{m}}$. It is thus possible to use galaxy cluster catalogs to constraint cosmological parameters (Vanderlinde et al. 2010; Sehgal et al. 2011; Planck Collaboration XX 2014) through a halo mass-function formalism.

We now have access to a full sky coverage for both X-ray emission with the ROSAT all sky survey (RASS) and tSZ emission with Planck (Planck Collaboration VIII 2011). Consequently, beyond tSZ clusters catalogs analysis, it is possible to perform tSZ angular power spectrum analysis. This process allows to consider all clusters on the covered sky without any selection function (see, e.g., Planck Collaboration VIII 2011), contrary to catalog-based analysis. This allows to catch the signals from higher redshift and lower mass objects that are not detected individually. Such measurement is limited by the contamination produced by other astrophysical components, mainly the cosmic infrared background (CIB, Puget et al. 1996; Fixsen et al. 1998).

It is difficult to perform the same power spectrum analysis with X-ray surveys. The X-ray photons, at low energy $(<0.5 \mathrm{keV})$, are absorbed by neutral hydrogen of our Galaxy and, at higher energy, the X-ray sky power is dominated by the emission from active galactic nuclei (AGN). Consequently, X-ray surveys are most commonly used to constraint the AGN spatial clustering (Krumpe et al. 2010; Miyaji et al. 2011; Krumpe et al. 2012).

In addition to auto-correlation power spectrum analysis, the cross power spectrum between tSZ effect and X-ray emission can be used. This cross-correlation is one of the easiest correlations to study, since both signals are produced by the same hot gas of electrons. Using such an approach allows the contamination to be minimized by other astrophysical components and suppresses the instrumental noise contribution to the power spectrum.

The tSZ-X cross spectrum is sensitive to both X-ray and tSZ scaling relations (see, e.g, Benson et al. 2013; Planck Collaboration XI 2011; Arnaud et al. 2010, for present constraints on scaling relations). This sensitivity limits the determination of cosmological parameters. However, it offers another possibility for constraining tSZ and X-ray scaling laws.

The utilization of the tSZ-X correlation has already been discussed in the literature. Diego et al. (2003) has attempted to directly compare theoretical predictions with the measured cross 
power spectrum between WMAP temperature anisotropy maps and ROSAT All Sky Survey (RASS). The limited sensitivity and resolution of the WMAP experiment leads to upper limits on the tSZ-X correlation. More recently, Hajian et al. (2013) have performed the measurement of the cross-correlation between the tSZ sky and an X-ray based catalog of clusters. From their analysis they derive $\sigma_{8}\left(\Omega_{\mathrm{m}} / 0.30\right)^{0.26}=0.80 \pm 0.02$.

We present an up-to-date modeling of the tSZ/X-ray crosscorrelation. In Sect. 2, we present our modeling of the tSZ-X cross-correlation. We give particular attention to the distribution in mass and redshift of the tSZ-X power. In Sect. 3, we explore the variations in the $\mathrm{tSZ}-\mathrm{X}$ spectrum with respect to cosmological and scaling-law parameters. We also discuss modeling uncertainties considering our knowledge on cosmological and scaling law parameters and present the main limitations for the tSZ$\mathrm{X}$ correlation measurement using simulated Planck-like data. Finally in Sect. 4, we predict the expected signal-to-noise for the tSZ-X correlation from simulations of Planck-like tSZ survey and ROSAT All Sky Survey, and we present the associated constraints on cosmological, scaling-law, and profile parameters.

Throughout the paper, we used the Planck-CMB best-fitting cosmology (Planck Collaboration XVI 2014) as our fiducial cosmological model, unless otherwise specified. Thus, we consider $H_{0}=67.1 \pm 1.4 \mathrm{~km} / \mathrm{s} / \mathrm{Mpc}, \sigma_{8}=0.834 \pm 0.027$, and $\Omega_{\mathrm{m}}=0.317 \pm 0.020$.

\section{Modeling tSZ-Xray cross-correlation}

\subsection{The $t S Z$ effect from galaxy clusters}

The tSZ effect consists of a small spectral distortion of the CMB black body (Sunyaev \& Zeldovich 1969, 1972), and its intensity is related to the integral of the pressure across the line of sight via the Compton parameter. This parameter in a given direction of the sky reads as

$y=\int \frac{k_{\mathrm{B}} \sigma_{\mathrm{T}}}{m_{\mathrm{e}} c^{2}} n_{\mathrm{e}} T_{\mathrm{e}} \mathrm{d} l$,

where $\mathrm{d} l$ is the distance along the line-of-sight, and $n_{\mathrm{e}}$ and $T_{\mathrm{e}}$ are the electron number density and the temperature, respectively.

In units of $\mathrm{CMB}$ temperature, the contribution of the tSZ effect to the submillimeter sky intensity for a given observation frequency $v$ is given by $\frac{\Delta T_{\mathrm{CMB}}}{T_{\mathrm{CMB}}}=g(v) y$. Neglecting relativistic corrections, we have $g(v)=\left[x \operatorname{coth}\left(\frac{x}{2}\right)-4\right]$, with $x=$ $h v /\left(k_{\mathrm{B}} T_{\mathrm{CMB}}\right)$. This function is equal to 0 around $217 \mathrm{GHz}$ and is negative at lower frequencies and positive for higher frequencies. Thus the spectral distortion induced by the hot gas of baryons provides a characteristic signal allowing the pressure distribution to be directly measured in galaxy clusters. In the context of a $\Lambda \mathrm{CDM}$ cosmology, this spectral distortion is known to be independent of the redshift. This has been tested and validated for a redshift range from 0 to 1 (Hurier et al. 2014). This characteristic spectral distortion can be used to separate the tSZ from other emissions of the microwave sky to derive Compton parameter map (see, e.g, Remazeilles et al. 2011; Hurier et al. 2013; Planck Collaboration VIII 2011).

\subsection{The $X$-ray emission from galaxy clusters}

The ionized gas in the intracluster medium produces an X-ray emission via Bremsstrahlung. This radiation is proportional to the square of the electronic density. The energy spectrum of the
X-ray emission from galaxy clusters depends mainly on the temperature, $T_{500}$, of the intracluster medium and, to a lesser extent on the metallicity, $Z$, of the gas. To model the metallicity evolution history, we follow Andreon (2012) using the relation derived from the analysis of 130 galaxy clusters in a redshift range from 0.1 to 1.3 ,

$\bar{Z}=\frac{0.35}{1-\exp (-11 / 6)}(1-\exp (-t(z) / 6))$

with $t(z)$ the age of the universe at a redshift $z$.

From an observational point of view, the X-ray spectrum depends on the redshift, $z$. X-ray photons are also absorbed by the neutral hydrogen in our Galaxy. This absorption is particularly significant for photons with $E<0.5 \mathrm{keV}$. Consequently, the observed count rate depends on the column density of neutral hydrogen, $n_{\mathrm{H}}$, on the line of sight.

To estimate the X-ray flux from each cluster, we computed an unabsorbed X-ray spectrum, $\phi_{\text {unabs }}=\mathrm{d} n_{\gamma} / \mathrm{d} E$, with $n_{\gamma}$ the number of emitted photons by the cluster inside a radius of $R_{500}$, using a MEKAL model (Mewe et al. 1985). To do this, we used a relation between the physical properties of the cluster, mass, and redshift and the temperature. These relations are presented in Sect. 2.5. Then, we computed the absorbed spectrum, $\phi_{\mathrm{abs}}$, as

$\phi_{\text {abs }}(E)=\phi_{\text {unabs }}(E) \exp \left[-n_{\mathrm{H}} \sigma(E)\right]$,

with $\sigma(E)$ the photoelectric cross section. The unabsorbed luminosity, $L_{500}$, in a given energy bin $\left[E_{\min }, E_{\max }\right]$ of a cluster reads as

$L_{500}=\int_{E_{\min }}^{E_{\max }} \mathrm{d} E E \phi_{\mathrm{abs}}(E)$.

Finally, the expected number count in a given energy bin $\left[E_{\min }^{\prime}, E_{\max }^{\prime}\right]$ is computed as

$S_{500}=\frac{a}{4 \pi \chi^{2}(z)} \int_{E_{\min }^{\prime}}^{E_{\max }^{\prime}} \mathrm{d} E^{\prime} \int_{0}^{\infty} \mathrm{d} E \mathcal{M}\left(E^{\prime}, E\right) A(E) \phi_{\mathrm{abs}}(E)$,

where $\chi(z)$ is the comoving angular distance at redshift $z, a$ the scale factor, $A(E)$ the effective area of the detector as a function of the energy, $\mathcal{M}\left(E^{\prime}, E\right)$ is the energy redistribution matrix, $E$ and $E^{\prime}$ are the photon energy ${ }^{1}$ and the measured value of photon energy, respectively.

We define the flux to count-rate conversion factor as $C R\left(z, Z, n_{\mathrm{H}}, T_{500}\right)=S_{500} / L_{500}$. However, clusters can be located at any position on the sky, so we have to convolve the $C R$ factor by the distribution of $n_{\mathrm{H}}$ on the sky:

$\overline{C R}\left(z, Z, T_{500}\right)=\int \mathrm{d} n_{\mathrm{H}} \mathcal{P}\left(n_{\mathrm{H}}\right) C R\left(z, Z, n_{\mathrm{H}}, T_{500}\right)$,

where $\mathcal{P}\left(n_{\mathrm{H}}\right)$ is the probability to have a column density of hydrogen $n_{\mathrm{H}}$ on the line of sight.

\subsection{The tSZ-Xray cross power spectra}

Decomposing both the tSZ Compton parameter map and X-ray count-rate map, we define

$y(\boldsymbol{n})=\sum_{\ell m} y_{\ell m} Y_{\ell m}(\boldsymbol{n})$,
$x(\boldsymbol{n})=\sum_{\ell m} x_{\ell m} Y_{\ell m}(\boldsymbol{n})$.

1 The values of $E_{\min }^{\prime}$ and $E_{\max }^{\prime}$ can differ from the values of $E_{\min }$ and $E_{\max }$. 
Thus, the power spectra of both tSZ effect and X-ray can be written as

$$
\begin{aligned}
C_{\ell}^{y y} & =\frac{1}{2 \ell+1} \sum_{m} y_{\ell m} y_{\ell m}^{*} \\
C_{\ell}^{x x} & =\frac{1}{2 \ell+1} \sum_{m} x_{\ell m} x_{\ell m}^{*},
\end{aligned}
$$

and the angular cross power spectrum of tSZ effect and X-ray count-rate map reads as

$$
C_{\ell}^{y x}=\frac{1}{2 \ell+1} \sum_{m} \frac{1}{2}\left(y_{\ell m} x_{\ell m}^{*}+y_{\ell m}^{*} x_{\ell m}\right) \text {. }
$$

To model this cross-correlation or the auto correlation power spectra, we assume the following general expression

$C_{\ell}=C_{\ell}^{1 \mathrm{~h}}+C_{\ell}^{2 \mathrm{~h}}+C_{\ell}^{\mathrm{diff}}$,

where $C_{\ell}^{1 \mathrm{~h}}$ is the Poissonian contribution, $C_{\ell}^{2 \mathrm{~h}}$ is the two-halo term that accounts for correlation in the spatial distribution of clusters over the sky, and $C_{\ell}^{\text {diff }}$ is produced by the warm-hot intergalactic medium (WHIM). In the following, considering the low density and the low temperature of the WHIM, we assume $C_{\ell}^{\mathrm{diff}}\left\langle\left\langle C_{\ell}^{1 \mathrm{~h}}+C_{\ell}^{2 \mathrm{~h}}\right.\right.$, and thus we neglect his contribution to the total power spectrum.

The Poissonian term can be computed by assuming the Fourier transform of tSZ and X-ray projected profiles weighted by the mass function, presented in Sect. 2.4, and the fluxes for tSZ effect and X-ray count rate (see, e.g, Komatsu \& Seljak 2002, for a derivation of the tSZ angular power spectrum):

$C_{\ell}^{y x, 1 \mathrm{~h}}=4 \pi \int \mathrm{d} z \frac{\mathrm{d} V}{\mathrm{~d} z \mathrm{~d} \Omega} \int \mathrm{d} M \frac{\mathrm{d}^{2} \mathrm{~N}}{\mathrm{~d} M \mathrm{~d} V}\left(1+\rho_{Y L} \sigma_{\log Y} \sigma_{\log L}\right) Y_{500} S_{500} y_{\ell} x_{\ell}$,

where $S_{500}=\overline{C R}\left(z, Z, T_{500}\right) L_{500}, \overline{C R}\left(z, Z, T_{500}\right)$ the flux to countrate conversion factor described in Sect. 2.2, $\frac{\mathrm{d}^{2} \mathrm{~N}}{\mathrm{~d} M \mathrm{~d} V}$ the clusters mass function described in Sect. 2.4, and $\frac{\mathrm{d} V}{\mathrm{~d} z \Omega}$ the element of comoving volume. The term $\left(1+\rho_{Y L} \sigma_{\log Y} \sigma_{\log L}\right)$ accounts for extra power produced by the scatter in the scaling relations described at Sect. 2.5.

The Fourier transform of the 3D profile projected across the line of sight on the sphere reads as

$p_{\ell}=\frac{4 \pi r_{\mathrm{o}}}{l_{\mathrm{s}}^{2}} \int_{0}^{\infty} \mathrm{d} r_{\mathrm{s}} r_{\mathrm{s}}^{2} p\left(r_{\mathrm{s}}\right) \frac{\sin \left(\ell r_{\mathrm{s}} / \ell_{\mathrm{s}}\right)}{\ell r_{\mathrm{s}} / \ell_{\mathrm{s}}}$

where $p\left(r_{\mathrm{s}}\right)$ is either the tSZ 3D profile or the X-ray count-rate $3 \mathrm{D}$ profile, and $r_{\mathrm{s}}=r / r_{\mathrm{o}}, \ell_{\mathrm{s}}=D_{\mathrm{A}}(z) / r_{\mathrm{o}}, r_{\mathrm{o}}$ is the scale radius of the profile.

The contribution of the two-halo term corresponds to large scale fluctuations in the matter power spectrum, which induce correlation in the cluster distribution over the sky. It can be computed as (see, e.g, Taburet et al. 2011, and references therein)

$$
\begin{aligned}
C_{\ell}^{y x, 2 \mathrm{~h}}= & 4 \pi \int \mathrm{d} z \frac{\mathrm{d} V}{\mathrm{~d} z \mathrm{~d} \Omega}\left(\int \mathrm{d} M \frac{\mathrm{d}^{2} \mathrm{~N}}{\mathrm{~d} M \mathrm{~d} V} Y_{500} y_{\ell} b_{\operatorname{lin}}(M, z)\right) \\
& \times\left(\int \mathrm{d} M \frac{\mathrm{d}^{2} \mathrm{~N}}{\mathrm{~d} M \mathrm{~d} V} S_{500} x_{\ell} b_{\operatorname{lin}}(M, z)\right) P(k, z)
\end{aligned}
$$

with $P(k, z)$ the matter power spectrum computed using $C L A S S$ (Lesgourgues 2011), and $b_{\text {lin }}(M, z)$ the time dependent linear bias factor that relates $P(k, z)$ to the power spectrum of the cluster distribution over the sky. Following Mo \& White (1996) and Komatsu \& Kitayama (1999), we adopt $b_{\text {lin }}(M, z)=1+$ $\left(v^{2}(M, z)-1\right) / \delta_{\mathrm{c}}(z)$, with $v(M, z)=\delta_{\mathrm{c}}(z) /\left[D_{\mathrm{g}}(z) \sigma(M)\right], D_{\mathrm{g}}(z)$ is the linear growth factor, and $\delta_{\mathrm{c}}(z)$ is the overdensity threshold for spherical collapse.

\subsection{Mass function}

Our computation of the tSZ-X correlation assumes the mass function calibrated on a numerical simulation from Tinker et al. (2008):

$\frac{\mathrm{d} N}{\mathrm{~d} M_{500} \mathrm{~d} V}=f(\sigma) \frac{\rho_{\mathrm{m}}(z=0)}{M_{500}} \frac{\mathrm{d} \ln \sigma^{-1}}{\mathrm{~d} M_{500}}$,

with

$f(\sigma)=A_{0}\left[1+\left(\frac{\sigma}{A_{1}}\right)^{A_{2}}\right] \exp \left(-\frac{A_{3}}{\sigma^{2}}\right)$,

and $\rho_{\mathrm{m}}(z=0)$ the mean matter density today. The coefficients $A_{0}, A_{1}, A_{2}$, and $A_{3}$ are given in Tinker et al. (2008) for various overdensities, $\Delta_{\text {mean }}$, with respect to the redshift-dependent mean cosmic density. These coefficients are interpolated to match $\Delta_{\text {critical }}$ defined with respect to the critical density. The relation between $\Delta_{\text {critical }}$ and $\Delta_{\text {mean }}$ is given by $\Delta_{\text {mean }}=\Delta_{\text {critical }} / \Omega_{\mathrm{m}}(z)$, with $\Omega_{\mathrm{m}}(z)$ the matter density parameter at redshift $z$. The standard deviation of the density perturbation in a sphere or radius $R, \sigma$, is computed in linear perturbation theory.

\section{5. $t S Z$ and $X$-ray fluxes}

A key step in the modeling of the cross-correlation between tSZ and X-ray is to relate the mass, $M_{500}$, and the redshift, $z$, of a given cluster to tSZ flux, $Y_{500}$, and X-ray luminosity $L_{500}$ in the $\left[E_{\min }-E_{\max }\right] \mathrm{keV}$ energy band ${ }^{2}$ in the rest frame of the cluster. The cross-correlation between $\mathrm{tSZ}$ effect and X-ray emission is thus highly dependent on the $M_{500}-Y_{500}$ and the $M_{500}-L_{500}$ relations in terms of normalization and slope. Consequently, we need to use the relations derived from a representative sample of galaxy clusters, with careful propagation of statistical and systematic uncertainties. We stress that for power spectrum analysis, the intrinsic scatter of such scaling laws has to be considered, because it will produce extra power that has to be accounted for in order to avoid biases.

We used the $M_{500}-Y_{500}$ scaling laws presented in Planck Collaboration XX (2014),

$E^{-\beta_{\mathrm{sz}}}(z)\left[\frac{D_{\mathrm{ang}}^{2}(z) Y_{500}}{10^{-4} \mathrm{Mpc}^{2}}\right]=Y_{\star}\left[\frac{h}{0.7}\right]^{-2+\alpha_{\mathrm{sz}}}\left[\frac{(1-b) M_{500}}{6 \times 10^{14} M_{\odot}}\right]^{\alpha_{\mathrm{sz}}}$,

with $E(z)=\Omega_{\mathrm{m}}(1+z)^{3}+\Omega_{\Lambda}$. The coefficients $Y_{\star}, \alpha_{\mathrm{sz}}$, and $\beta_{\mathrm{sz}}$ from Planck Collaboration XX (2014), are given in Table 1. We used $b=0.2$ for the bias between X-ray estimated mass and effective mass of the clusters. To model the $L_{500}-M_{500}$ relation, we used the relation derived by Arnaud et al. (2010) from the REXCESS sample (Böhringer et al. 2007):

$[h E(z)]^{-\beta_{\star}}\left(\frac{L_{500}}{10^{44} \mathrm{erg} \mathrm{s}^{-1}}\right)=L_{\star}\left[\frac{M_{\mathrm{x}, 500}}{3 \times 10^{14} M_{\odot}}\right]^{\alpha_{\mathrm{x}}}$,

where $M_{\mathrm{x}, 500}$ is the cluster mass estimated from X-ray observations. It is related to the true mass $M_{500}$ through $M_{\mathrm{x}, 500}=$ $(1-b) M_{500}$. The coefficients $L_{\star}, \alpha_{\mathrm{x}}$, and $\beta_{\mathrm{x}}$ are given in Table 1 .

The evolution of the $M_{500}-L_{500}$ relation suffers from large uncertainties (Reichert et al. 2011). Consequently, we fixed $\beta_{\mathrm{x}}$

2 By convention, the energy range [0.1-2.4] $\mathrm{keV}$ is used for the definition of the $M_{500}-L_{500}$ scaling relation. 
Table 1. Scaling-law parameters and error budget for both $Y_{500}-M_{500}$ (Planck Collaboration XX 2014), $L_{500}-M_{500}$ (Arnaud et al. 2010), and $Y_{500}-T_{500}$ (Planck Collaboration XX 2014) relations.

\begin{tabular}{cc|cc|cc}
\hline \hline \multicolumn{2}{c}{$M_{500}-Y_{500}$} & \multicolumn{2}{c}{$M_{500}-L_{500}$} & \multicolumn{2}{c}{$M_{500}-T_{500}$} \\
\hline $\log Y_{\star}$ & $-0.19 \pm 0.02$ & $\log L_{\star}$ & $0.724 \pm 0.032$ & $\log T_{\star}$ & $-4.27 \pm 0.02$ \\
$\alpha_{\mathrm{sz}}$ & $1.79 \pm 0.08$ & $\alpha_{\mathrm{x}}$ & $1.64 \pm 0.12$ & $\alpha_{\mathrm{T}}$ & $2.85 \pm 0.18$ \\
$\beta_{\mathrm{sz}}$ & $0.66 \pm 0.50$ & $\beta_{\mathrm{x}}$ & $7 / 3$ & $\beta_{\mathrm{T}}$ & 1 \\
$\sigma_{\log Y}$ & $0.075 \pm 0.010$ & $\sigma_{\log L}$ & $0.183 \pm 0.032$ & $\sigma_{\log T}$ & $0.14 \pm 0.02$ \\
\hline
\end{tabular}

to a self-similar value of $7 / 3$. However, we verified that variations from 2 to 3 do not change our results with respect to other sources of uncertainties on the $M_{500}-L_{500}$ relation. Indeed, X-ray signal is dominated by low redshift clusters, and thus presents small variations with the redshift evolution of the $M_{500}-L_{500}$ relation.

The two relations, $M_{500}-Y_{500}$ and $M_{500}-L_{500}$, have intrinsic scatters, $\sigma_{\log Y}=0.075$ and $\sigma_{\log L}=0.183$, respectively. These scatters will contribute to the total power measured on the sky. Indeed, the quantity $\left\langle Y^{2}{ }_{500}\right\rangle$ is equal to $\left(1+\sigma_{\log Y}^{2}\right) Y_{500}^{2}$, and $\left\langle L^{2}{ }_{500}\right\rangle$ is equal to $\left(1+\sigma_{\log L}^{2}\right) L_{500}^{2}$. The cross-correlation power spectrum of tSZ and X-ray can be affected by the same effect. However, for a cross-correlation this scatter bias depends on the correlation between the scatters of the $M_{500}-Y_{500}$ and the $M_{500}-L_{500}$ scaling relations. This question is addressed in Sect. 3.2.

We also need to have an estimate of the cluster temperature, $T_{500}$. In this work, we used the scaling law from Planck Collaboration XI (2011):

$E(z)^{-\beta_{\mathrm{T}}} Y_{500}=T_{\star}\left[\frac{T_{500}}{6 \mathrm{keV}}\right]^{\alpha_{\mathrm{T}}}$

where the coefficients $T_{\star}, \alpha_{\mathrm{T}}$ and $\beta_{\mathrm{T}}$ are given in Table 1 . This relation also presents an intrinsic scatter $\sigma_{\log T}=0.14 \pm 0.02$. We verified that this scatter has no significant impact on the tSZ-X cross power-spectrum amplitude with respect to the scatter from $M_{500}-Y_{500}$ and $M_{500}-L_{500}$ relations.

\subsection{Pressure and density profiles}

The tSZ effect is directly proportional to the pressure integrated across the line of sight. In this work, we model the galaxy cluster pressure profile by a generalized Navarro Frenk and White (GNFW, Navarro et al. 1997; Nagai et al. 2007) profile of the form

$\mathbb{P}(r)=\frac{P_{0}}{\left(c_{500} r\right)^{\gamma}\left[1+\left(c_{500} r\right)^{\alpha}\right]^{(\beta-\gamma) / \alpha}}$.

For the parameters $c_{500}, \alpha, \beta$, and $\gamma$, we used the best-fitting values from Arnaud et al. (2010) presented in Table 1. The absolute normalization of the profile $P_{0}$ is set assuming the scaling laws $Y_{500}-M_{500}$ presented in Sect. 2.5.

To model the X-ray emission, we need both the density, $n_{\mathrm{e}}(r)$, and the temperature, $T_{\mathrm{e}}(r)$, profiles. Thus, we assume a polytropic equation of state (see, e.g., Komatsu \& Seljak 2001), $\mathbb{P}(r)=n_{\mathrm{e}}(r) T_{\mathrm{e}}(r)$, with $n_{\mathrm{e}}(r) \propto T_{\mathrm{e}}(r)^{\delta}$ where $\delta$ is the polytropic index. Considering that the X-ray emission varies with $T_{\mathrm{e}}^{0.5}$ and $n_{e}^{2}$, the X-ray brightness profile is proportional to $P(r)^{\epsilon_{p}}$, where $\epsilon_{p}=\frac{2 \delta}{\delta+1}+\frac{1}{2 \delta}$ ranges from 1.5 to 2.0 for $1.0<\delta<\infty$.

For the X-ray flux to count-rate conversion factor, we only assume an averaged temperature $T_{500}$, and then the X-ray flux is directly proportional to $n_{\mathrm{e}}^{2}(x)$. The overall normalization of the $\mathrm{X}$-ray emission profile is deduced from the scaling law $L_{500}-$ $M_{500}$ presented in Sect. 2.5.

\section{Results}

\subsection{The $t S Z-X$ power spectrum}

In Fig. 1, we present the angular cross power spectrum between tSZ and X-rays (assuming a $0.5-2.0 \mathrm{keV}$ energy band for the ROSAT experiment). The power spectrum is predicted for our fiducial cosmological model. We observe that the tSZ-X power spectrum is dominated by the one-halo term throughout the entire range of multipole, from $\ell=0$ to $\ell=10000$. For low multipoles $(\ell<1000)$, the tSZ-X power spectrum follows a power law $C_{\ell}^{y x} \propto \ell^{-1.1}$.

The correlation factor between $\mathrm{tSZ}$ and X-ray surveys, $\rho_{y x}=$ $\frac{C_{\ell}^{y, x}}{\sqrt{C_{\ell}^{y y} C_{\ell}^{x x}}}$, as a function of $\ell$ presents a slow variation from 0.8 at low $\ell$ to 0.6 at high $\ell$. The smaller correlation factor on small scales highlight the difference in slopes in $\mathrm{tSZ}$ and $\mathrm{X}$-ray profiles in the core of the cluster, as the tSZ profile decreases with $T_{\mathrm{e}} n_{\mathrm{e}}$ and the X-ray profile with $n_{\mathrm{e}}^{2}$.

\subsection{The scaling-relation scatter bias}

The amplitude of the one-halo term of tSZ-X power spectrum is sensitive to the scatter of scaling relations, which produce an excess of power. The two-halo term is not affected by the scatter. For the tSZ auto-correlation power spectrum, the scatter produces a negligible bias of $0.5 \%$. For the X-ray power spectrum, the effect reach $3.3 \%$.

The bias on tSZ-X cannot be estimated as easily. Indeed the correlation between $M_{500}-Y_{500}$ and $M_{500}-L_{500}$ scatters has to be known. The quantity $\left\langle Y_{500} L_{500}\right\rangle$ is equal to $(1+$ $\left.\rho_{Y L} \sigma_{\log Y} \sigma_{\log L}\right) Y_{500} L_{500}$, where $\rho_{Y L}$ is the correlation between the $M_{500}-Y_{500}$ and $M_{500}-L_{500}$ scatters. Consequently, the bias is zero for a 0 correlation and maximum for a full correlation.

Using the $Y_{500}-L_{500}$ scatter, $\sigma_{\log Y L}=0.14 \pm 0.02$ (Planck Collaboration XI 2011), it is possible to estimate $\rho_{Y L}$ through

$\rho_{Y L}=\frac{\sigma_{\log Y}^{2}\left(\frac{\alpha_{\mathrm{SZ}}}{\alpha_{\mathrm{X}}}\right)^{2}+\sigma_{\log L}^{2}-\sigma_{\log Y L}^{2}}{2 \frac{\alpha_{\mathrm{SZ}}}{\alpha_{\mathrm{X}}} \sigma_{\log Y} \sigma_{\log L}}=0.9 \pm 0.3$.

This finding is consistent with an almost full correlation between $M_{500}-Y_{500}$ and $M_{500}-L_{500}$ scatters. This value for $\rho_{Y L}$ leads to a bias of $1.4 \%$ for the amplitude of the tSZ-X power spectrum. Finally, we note that the $M_{500}-Y_{500}$ and $M_{500}-L_{500}$ scatters account for the profile scatter from cluster to cluster. 


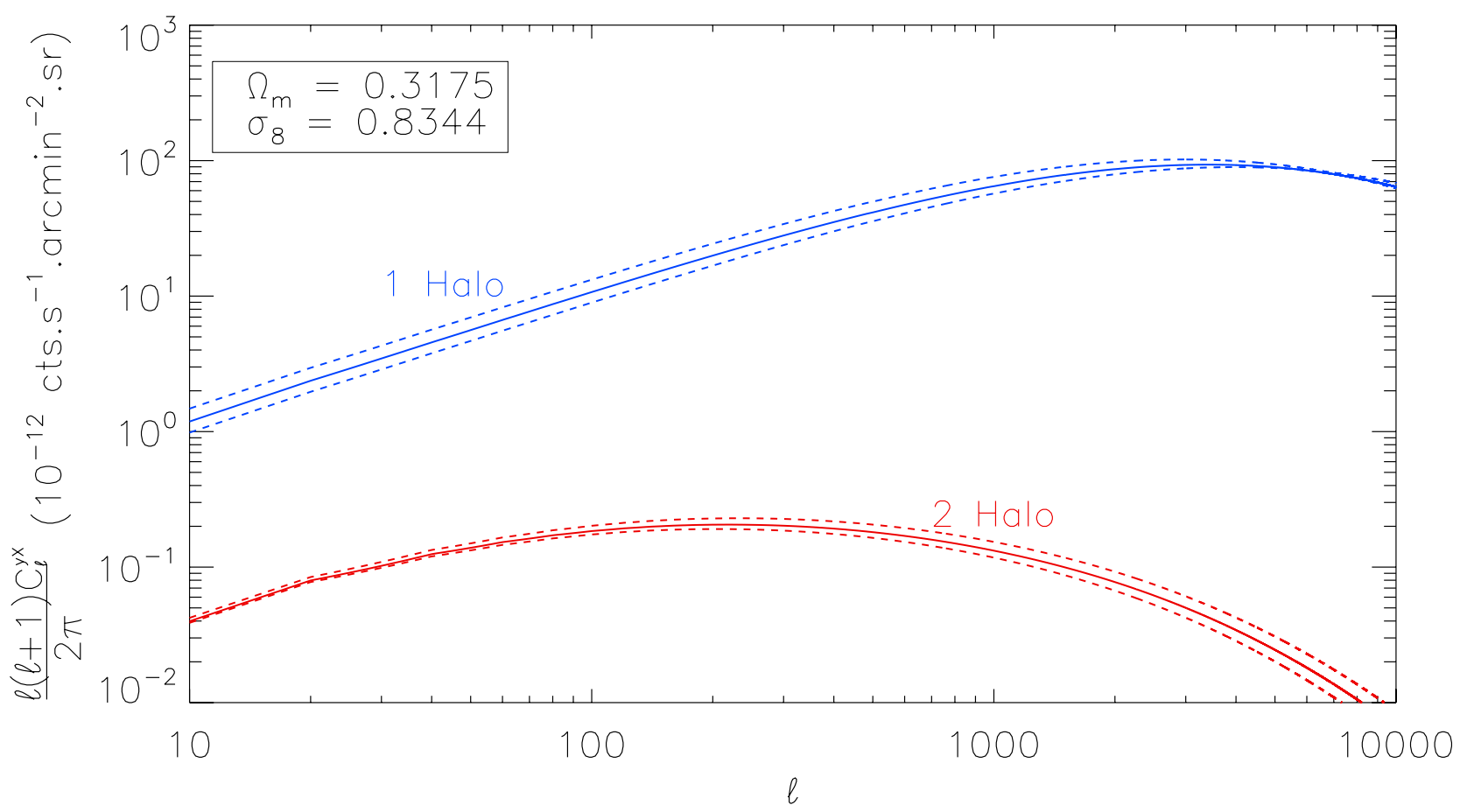

Fig. 1. tSZ-Xray cross-correlation for the 1-halo term in blue and for the 2-halo term in red, considering the ROSAT hard band from 0.5 to $2.0 \mathrm{keV}$. The dashed lines represent the $1 \sigma$ level considering variations in scaling-relation power laws. This cross-correlation spectrum has been predicted using our fiducial cosmological model.

\subsection{Redshift and mass distribution of the tSZ-Xray cross correlation}

In Figs. 2 and 3, we present the distribution of the tSZ-Xray cross correlation power spectrum as a function of the redshift and the mass for various values of $\ell$ from 20 to 10000 for our fiducial model. We observe that the power below $\ell=100$ is dominated by a local object at redshifts below 0.2 . Whereas at high multipole values, $\ell=10000$, we are sensitive to structures up to $z=1.5$. We observe that the small and large angular scales of the power spectrum sample distinct populations in terms of redshift.

Unlike the redshift distribution, we observe that the mass dependency presents small variations for $\ell$ ranging from 20 to 2000. For these multipoles, the power is dominated by objects with $M_{500}$ above $10^{14} M_{\odot}$. Lower mass objects only make significant contributions to the total power for a very high multipole value $\ell \simeq 10000$.

The mass function predicts a number of objects above a given mass $M_{500}$ that drastically increases when $M_{500}$ decreases. Similarly, the comoving volume increases with increasing redshift (below $z \simeq 2$ ). The two-halo term presented in Sect. 2.3 is proportional to the number of clusters, which contributes significantly to the total power, to the square, unlike the one-halo terms that are linearly related to this quantity.

This explains the relative small amplitude of the two-halo term with respect to the one-halo term for the tSZ-X power spectrum. Indeed, the low-redshift depth and the high mass sensitivity of the tSZ-X power spectrum imply that the total power is dominated by a small number of objects.

Consequently, low values of $\alpha_{\mathrm{x}}$ or $\alpha_{\mathrm{sz}}$ promote the two-halo term with respect to the one-halo term. The two-halo term becomes significant at low- $\ell$ for $\alpha_{\mathrm{x}}+\alpha_{\mathrm{sz}}<3$. However, such values are excluded by existing constraints on the $M_{500}-Y_{500}$ and the $M_{500}-L_{500}$ relations (see Table 1 for the allowed uncertainty range and Fig. 1 for the impact on tSZ-X cross power spectrum of these uncertainties).

\section{4. $t S Z-X$ cross-correlation dependencies with modeling parameters}

Our modeling is affected both by cosmological and scaling law parameters. First, we focus on cosmological parameters, with a particular attention to $H_{0}, \Omega_{\mathrm{m}}$, and $\sigma_{8}$. In Fig. 4, we present the variation in the tSZ-X cross spectrum as a function of $H_{0}$ from 60 to $80 \mathrm{~km} \mathrm{~s}^{-1} \mathrm{Mpc}^{-1}$ in steps of $1 \mathrm{~km} \mathrm{~s}^{-1} \mathrm{Mpc}^{-1}, \Omega_{\mathrm{m}}$ from 0.2 to 0.4 in steps of 0.01 , and $\sigma_{8}$ from 0.7 to 0.9 in steps of 0.01 . In the most general case, those variations depend on the multipole $\ell$, as presented in Figs. 2 and 3. Each multipole is sensitive to different regions of the mass function and thus present different sensitivities to the cosmological parameters:

$A_{\mathrm{cl}} \propto\left(\frac{H_{0}}{67}\right)^{\alpha_{\mathrm{H}}(\ell)}\left(\frac{\Omega_{\mathrm{m}}}{0.32}\right)^{\alpha_{\Omega}(\ell)}\left(\frac{\sigma_{8}}{0.83}\right)^{\alpha_{\sigma}(\ell)}$,

where $A_{\mathrm{cl}}$ is the amplitude of the tSZ-X power spectrum. However as shown in Fig. 4, we do not observe a significant distortion of the shape of the cross-correlation with a variation in the cosmological parameters.

Similar expressions can be used for tSZ and X-ray autocorrelation spectra. In Table 2 we present the values of $\alpha_{\mathrm{H}}(\ell)$, $\alpha_{\Omega}(\ell)$, and $\alpha_{\sigma}(\ell)$ for each spectra, tSZ-X, tSZ-auto, X-auto.

In Fig. 5, we present the power law index variations with respect to $\ell$. For the tSZ auto-correlation we observe $\alpha_{\mathrm{H}} \simeq 0$; however, both the X-ray auto-spectrum and the X-tSZ cross spectrum present significant variations with $H_{0}$, with $\alpha_{\mathrm{H}}^{X} \simeq 5$ and $\alpha_{\mathrm{H}}^{X S Z} \simeq 2.5$. These dependency variation are produced by the $L_{500}-M_{500}$ scaling relation. We also observe that $\alpha_{\Omega}$ ranges from 2.8 to 3.8 and $\alpha_{\sigma}$ from 7 to 9 . These variations in spectral indices with respect to $\ell$ are small. 


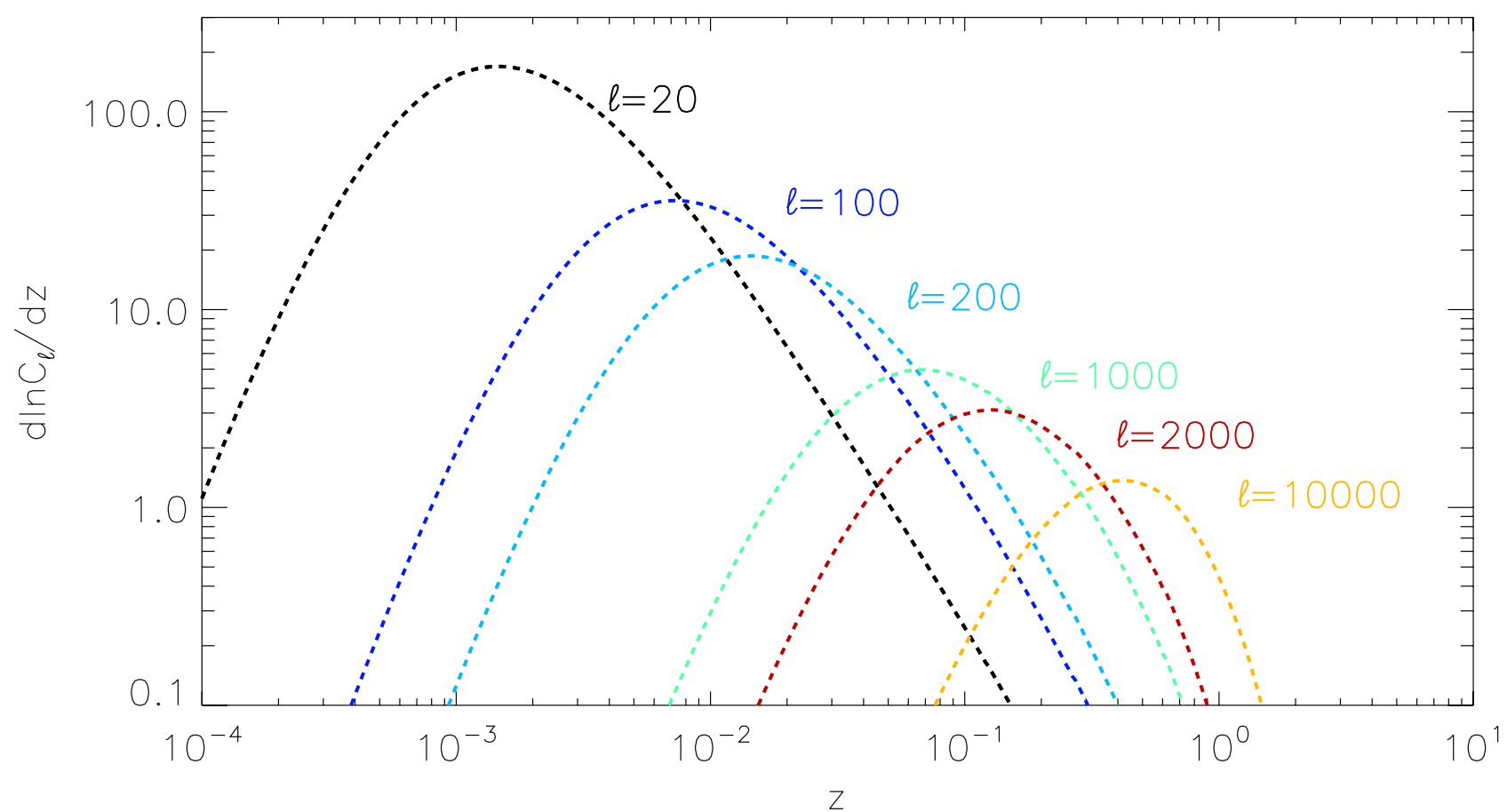

Fig. 2. Redshift distribution of the contribution to the total tSZ/Xray cross-correlation power for several values of $\ell$. In black for $\ell=20$, in dark blue for $\ell=100$, in light blue for $\ell=200$, in green for $\ell=1000$, in orange for $\ell=2000$, and in red for $\ell=10000$.

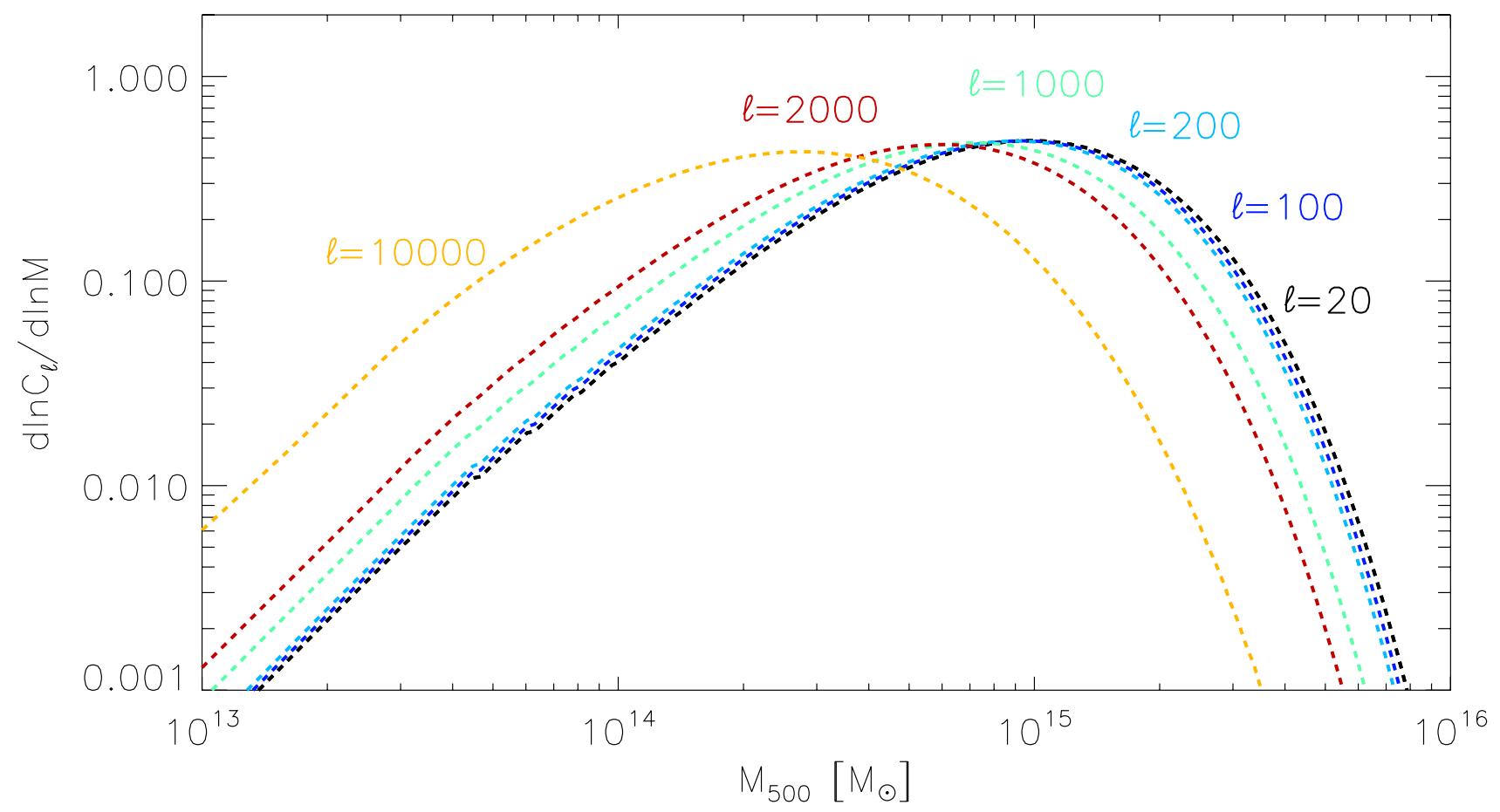

Fig. 3. Mass distribution of the contribution to the total tSZ/Xray cross-correlation power for several values of $\ell$. In black for $\ell=20$, in dark blue for $\ell=100$, in light blue for $\ell=200$, in green for $\ell=1000$, in orange for $\ell=2000$, and in red for $\ell=10000$.

In Table 2, we provide fitting formula for each power law index with respect to $\ell$ using the following parametric formula:

$\alpha=p_{1}+p_{2}\left(\ell+p_{3}\right)^{p_{4}}$.

Values for $p_{1}, p_{2}, p_{3}, p_{4}$ are provided in Table 2 . We note that the power law indices are also functions of the cosmological parameters. Consequently, we stress that the formula given above has been estimated for cosmological parameters $\left(H_{0}, \Omega_{\mathrm{m}}\right.$, $\left.\sigma_{8}\right)=(67,0.32,0.83)$, and thus can only be considered accurate for $10 \%$ variations around these parameters.

In addition to the sensitivity to cosmological parameters, the tSZ-X correlation is highly sensitive to the scaling relations described in Sect. 2.5 and cluster profiles described in Sect. 2.6, both of which are related. Consequently for the present work, we consider the profile from Arnaud et al. (2010) that has been used to estimate the $Y_{500}-M_{500}$ scaling relation. However, we verified 

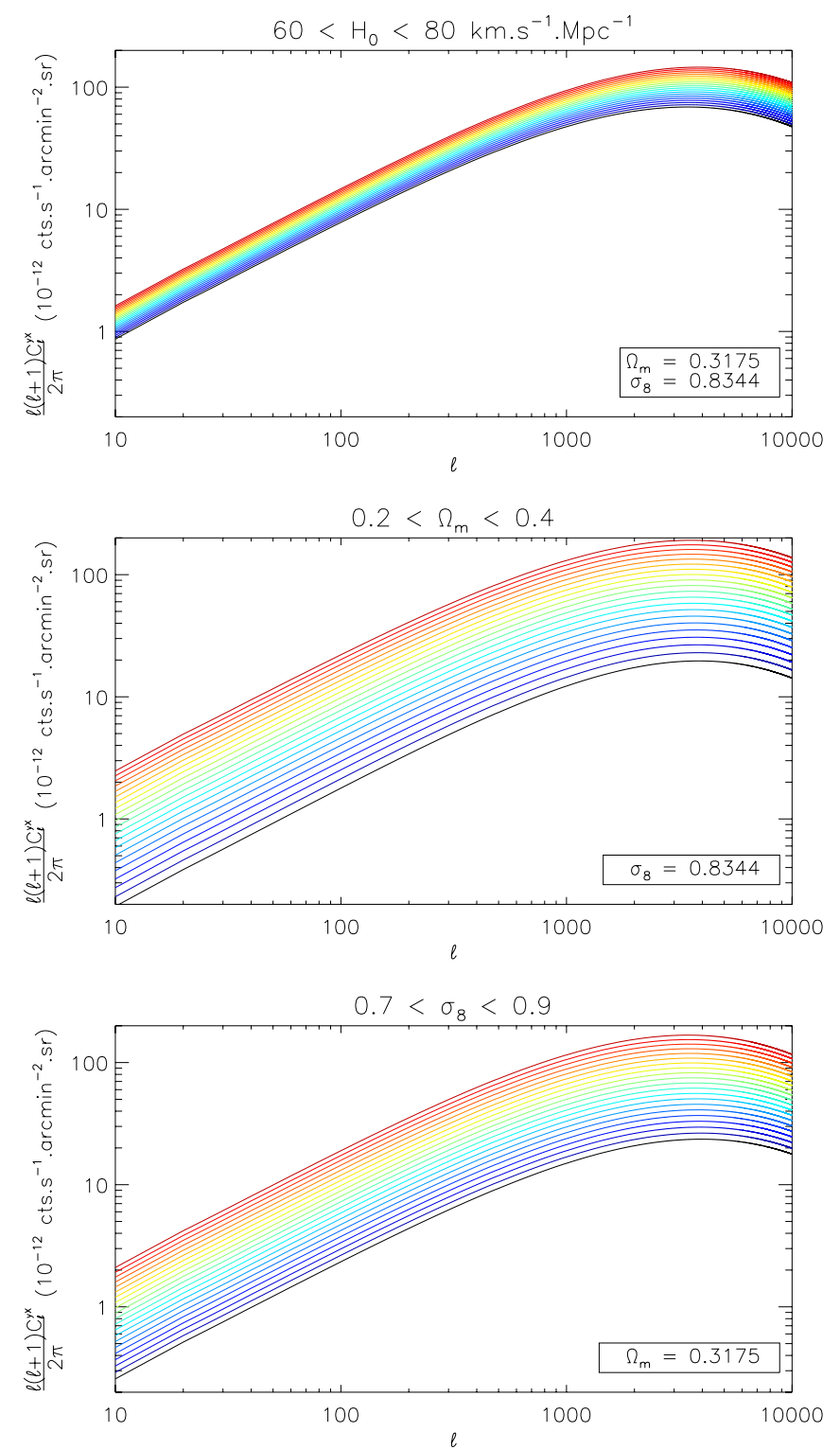

Fig. 4. Theoretical tSZ-Xray cross-correlation power spectra, as a function of, from top to bottom, $H_{0}, \Omega_{\mathrm{m}}$, and $\sigma_{8}$. From blue to red for 21 values of $H_{0}, \Omega_{\mathrm{m}}$, and $\sigma_{8}$, starting at $60 \mathrm{~km} \mathrm{~s}^{-1} \mathrm{Mpc}^{-1}, 0.2$, and 0.7 in steps of $1 \mathrm{~km} \mathrm{~s}^{-1} \mathrm{Mpc}^{-1}, 0.01$, and 0.01 , respectively.

that the tSZ-X cross spectrum varies by $10 \%$ at most for various pressure profiles, as Arnaud et al. (2010), Planck Collaboration Int. V (2013), and Sayers et al. (2013) and for various polytropic indices from 1 to $\infty$ (isothermal case).

A variation in the scaling-relation normalization translates into a variation in the amplitude of the cross spectrum. However, the scaling law power law indices will produce a modification of the shape of the tSZ-X correlation.

In the following, we model the deviation from our reference scaling laws presented in Sect. 2.5 as

$$
\begin{aligned}
\tilde{Y}_{500} & =Y_{500}\left(\frac{M_{500}}{3 \times 10^{14}}\right)^{\delta \alpha_{\mathrm{sz}}} \\
\tilde{L}_{500} & =L_{500}\left(\frac{M_{500}}{3 \times 10^{14}}\right)^{\delta \alpha_{\mathrm{x}}},
\end{aligned}
$$

Table 2. Power law indices of the tSZ-X, tSZ-auto, and X-auto power spectra for variations with from top to bottom $H_{0}, \Omega_{\mathrm{m}}$, and $\sigma_{8}$ as described in Eqs. (23) and (24).

\begin{tabular}{c|ccccc}
\hline \hline & $\overline{\alpha_{\mathrm{H}}}$ & $p_{1}$ & $p_{2}$ & $p_{3}$ & $p_{4}$ \\
\hline tSZ-X & 2.38 & -0.025 & 1.027 & 967 & 0.1114 \\
tSZ-auto & -0.29 & -3.60 & 1.50 & 597 & 0.1071 \\
X-auto & 5.09 & 0 & 3.475 & 1998 & -0.04747 \\
\hline & $\overline{\alpha_{\Omega}}$ & $p_{1}$ & $p_{2}$ & $p_{3}$ & $p_{4}$ \\
tSZ-X & 3.42 & 3.24 & $1.66 \times 10^{5}$ & 1426 & -1.767 \\
tSZ-auto & 3.14 & 2.836 & $7.99 \times 10^{7}$ & 1680 & -2.463 \\
X-auto & 3.50 & 3.42 & $1.66 \times 10^{5}$ & 1704 & -1.844 \\
\hline & $\overline{\alpha_{\sigma}}$ & $p_{1}$ & $p_{2}$ & $p_{3}$ & $p_{4}$ \\
tSZ-X & 8.12 & 0 & 12.55 & 1498 & -0.0557 \\
tSZ-auto & 8.87 & 0 & 15.06 & 2017 & -0.0660 \\
X-auto & 7.46 & 0 & 12.12 & 1190 & -0.0620 \\
\hline
\end{tabular}

where $\delta \alpha_{\mathrm{sz}}$ and $\delta \alpha_{\mathrm{x}}$ represent the deviations from the reference scaling law indices, $\alpha_{\mathrm{sz}}$ and $\alpha_{\mathrm{x}}$, for $M_{500}-Y_{500}$ and $M_{500}-L_{500}$, respectively.

In Fig. 6, we present the variation in the tSZ-X, X-ray, and tSZ power spectra with the scaling law indices, $\alpha_{\mathrm{sz}}$ and $\alpha_{\mathrm{x}}$, insteps of 0.025 for each index and 0.05 for their sum. We note that the tSZ-X power spectrum is only sensitive to the sum of the indices, $\alpha_{\mathrm{sz}}+\alpha_{\mathrm{x}}$. In terms of amplitude, the tSZ-X power spectrum follows

$A_{\mathrm{cl}} \propto\left(\frac{Y_{\star}}{0.65} \frac{L_{\star}}{1.88}\right)\left(\frac{1-b}{0.8}\right)^{\alpha_{\mathrm{sz}}+\alpha_{\mathrm{x}}}$.

We observe in Fig. 6 that increasing the value of $\alpha_{\mathrm{sz}}$ and $\alpha_{\mathrm{x}}$ increases the power at low- $\ell$ where high-mass objets dominate the signal and decreases the power at high $\ell$, that is dominated by low-mass objects. However, the shape distortion of the power spectra occurs at high- $\ell$. The impact of scaling law indices start to be significant at $\ell>1000, \ell>800$, and $\ell>2000$ for tSZ$X, \mathrm{tSZ}$, and $\mathrm{X}$ power spectra, respectively. We observe that the $\mathrm{X}$-ray power spectrum presents the lowest sensitivity in terms of shape with respect to $\delta \alpha_{\mathrm{x}}$.

We can infer a global dependence of the tSZ-X crosscorrelation amplitude for fixed scaling-law indices and fixed profiles,

$A_{\mathrm{cl}}=\left(\frac{\sigma_{8}}{0.83}\right)^{8.12}\left(\frac{\Omega_{\mathrm{m}}}{0.32}\right)^{3.42}\left(\frac{H_{0}}{67}\right)^{2.38}\left(\frac{Y_{\star}}{0.65} \frac{L_{\star}}{1.88}\right)\left(\frac{1-b}{0.8}\right)^{\alpha_{\mathrm{sz}}+\alpha_{\mathrm{x}}}\left(\frac{N}{N_{0}}\right)$,

where $N$ is a normalization parameter for the mass function.

\subsection{Modeling uncertainties}

Uncertainties on the predicted spectrum are produced by uncertainties on galaxies cluster properties and uncertainties on the cosmology. We used the uncertainties on cluster properties listed in Table 1 and propagated them to predict the power spectra. For the mass function, we assumed an overall uncertainty of $10 \%$ (Evrard et al. 2002) and for the bias, $b$, we assumed an uncertainty of $10 \%$ (e.g, Piffaretti \& Valdarnini 2008). For the uncertainties on cosmological parameters, we consider two different sets of cosmological parameters, our fiducial model named cosmo 1, and a second model based on the best fit from Planck Collaboration XX (2014) named cosmo 2. For both sets of parameter, we propagated the uncertainties to the tSZ-X power spectra. We carefully account for correlation between uncertainties between parameters for each set. 
Table 3. Modeling error budget for the tSZ-X cross spectrum and the $\mathrm{X}$ and SZ auto spectra.

\begin{tabular}{cccccccccc}
\hline \hline Spectrum & $b$ & $Y_{\star}-L_{\star}$ & $\alpha_{\text {SZ,X }}$ & Profiles & $m f$ & Total cosmo fix & cosmo 1 & cosmo 2 & Total \\
\hline X-tSZ & $35 \%$ & $9 \%$ & $1-4 \%$ & $10 \%$ & $10 \%$ & $37 \%$ & $31 \%$ & $27 \%$ & $48 \%$ \\
tSZ-auto & $32 \%$ & $9 \%$ & $1-5 \%$ & $10 \%$ & $10 \%$ & $38 \%$ & $34 \%$ & $28 \%$ & $50 \%$ \\
X-auto & $37 \%$ & $16 \%$ & $2-4 \%$ & $10 \%$ & $10 \%$ & $37 \%$ & $26 \%$ & $26 \%$ & $48 \%$ \\
\hline
\end{tabular}

Notes. The total uncertainties are computed assuming cosmo 2. All uncertainties are expressed in percentage of the total power.
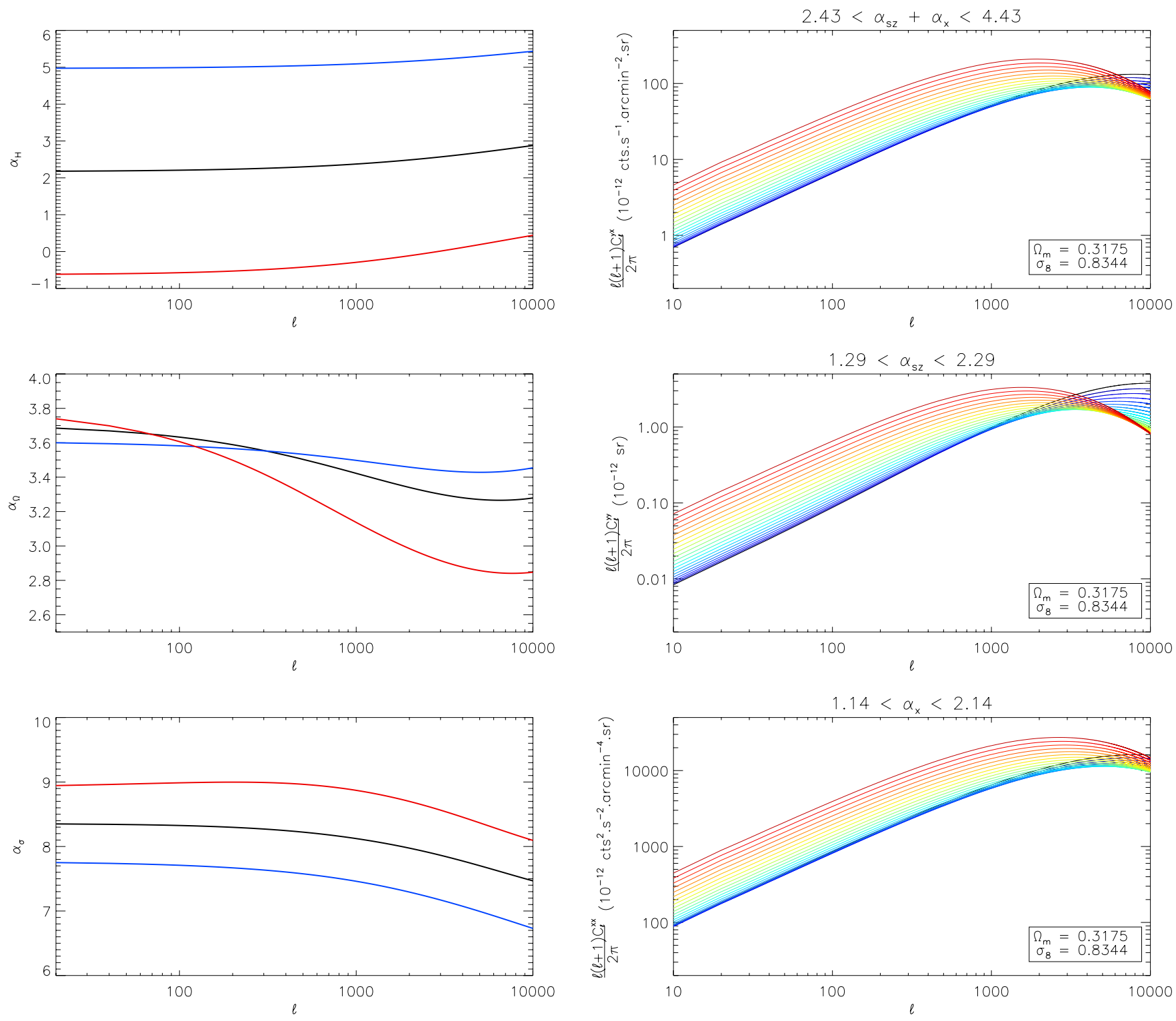

Fig. 5. Power law index variation as a function of $\ell$ for in black the $\mathrm{X}$-tSZ cross-correlation, in red the tSZ auto-correlation, and in blue the X-ray auto-correlation. From top to bottom for $\alpha_{\mathrm{H}}(\ell), \alpha_{\Omega}(\ell)$, and $\alpha_{\sigma}(\ell)$.

Fig. 6. From top to bottom: variation in the theoretical tSZ-X, tSZ-tSZ, and X-X power spectra as a function of $\alpha_{\mathrm{sz}}+\alpha_{\mathrm{x}}, \alpha_{\mathrm{sz}}$ and $\alpha_{\mathrm{x}}$, in steps of $0.05,0.025$, and 0.025 , respectively.

We notice that the propagation of the uncertainty on $\alpha_{\mathrm{SZ}, \mathrm{X}}$ depends on the multipole, consequently we provide a range for the uncertainty for $0<\ell<2000$.

In Table 3, we present the modeling uncertainties on the tSZ-X cross correlation. We stress that these uncertainties translate into an overall normalization of the spectrum. We also provide the uncertainty levels for the tSZ and X-ray auto correlation

spectra. Assuming a fixed cosmology, we noted that the error budget is limited by our knowledge of $b$, leading to an uncertainty of about $35 \%$ on the amplitude of the tSX power spectrum. If we propagate the uncertainties on cosmological parameters to the tSZ-X power spectrum, we derived an uncertainty of $31 \%$ for the cosmo 1 and an uncertainty of $27 \%$ for cosmo 2 . Cosmo 2 allows us to obtain slightly lower uncertainties for the 
tSZ-X prediction, because the degeneracy between cosmological parameters is similar for tSZ and tSZ-X spectra, unlike for cosmo 1 cosmology, which presents different degeneracies.

We finally note that for the $\mathrm{tSZ}-\mathrm{X}$ cross power spectrum, the error budget from cluster properties and cosmological parameters have the same order of magnitude. The total uncertainty that includes both contributions reaches $48 \%$.

\section{Prediction of the tSZ-Xray spectrum measurement}

To estimate the tSZ-Xray cross correlation, it is possible to use several approaches. One of them relies on the cross-correlation of frequency maps of the microwave sky with an X-ray map, see Sects. 4.3 and 4.4. Another one consists in using a recovered tSZ map (see, e.g., Hurier et al. 2013) and an X-ray map, see Sect. 4.5. In the following, we discuss the advantages and drawbacks of each approach.

Measurement of the tSZ-X correlation is limited by both instrumental characteristics and contaminating astrophysical emissions. To estimate the constraints that can be reached on the tSZ-X power spectrum, we predicted the expected signal-tonoise ratio assuming the Planck nominal mission characteristics (i.e., noise and beams Planck Collaboration I 2014) for the microwave observations and the RASS (Voges et al. 1999) for X-rays.

\subsection{The micro-wave and $X$-ray skies}

To simulate the sky emission at microwave frequencies with the appropriate level of noise, we used the Planck Sky Model (PSM, see Delabrouille et al. 2013, and references therein). At microwave frequencies, the main astrophysical emissions are the diffuse Galactic free-free, synchrotron, thermal dust emissions, the anomalous microwave emission, the emission from Galactic and extragalactic point sources, the CIB, the Zodiacal light emission, and the tSZ effect in clusters of galaxies.

To account for the signal from the X-ray sky, we used the RASS data in the energy range $[0.5,2.0] \mathrm{keV}$, degraded at an angular resolution of $F W H M=2^{\prime}$, and projected again in the HEALpix pixelization (Górski et al. 2005) following a nearest neighbor interpolation. There are several types of astrophysical objects that emit in the X-rays: extragalactic ones such as galaxy clusters, black holes in AGN, the combination of unresolved X-ray emitting objects producing the X-ray background (Freyberg et al. 1992), but also galactic sources, mainly supernova remnants and stars.

In addition to the signal from galaxy clusters, there are other astrophysical emissions that are also correlated between the X-ray and the microwave skies. This is the case of the radioloud AGNs and the CIB. Both AGN and CIB present a different frequency dependence than the tSZ effect and consequently can, in principle, be separated from the galaxy clusters' contribution to the tSZ-X cross correlation. In addition, AGNs are point-like at Planck and ROSAT angular resolutions, and thus can be separated from the clusters' contribution by the shape of the power spectrum.

The emission from our galaxy is also present on sky at microwave frequencies (synchrotron, Free-Free, and thermal dust) and in ROSAT energy bands $\left(n_{\mathrm{H}}\right.$ absorption and galactic X-ray emissions). However, considering only the ROSAT hard band $(0.5-2.0 \mathrm{keV})$ reduces the effect of the $n_{\mathrm{H}}$ absorption, and utilizing a galactic mask reduces the contamination by galactic foregrounds. As for the contamination by extragalactic point sources, such contamination will present a frequency dependence that differs from a tSZ spectrum, and thus can be distinguished using multifrequency analysis.

\subsection{Statistical and systematic uncertainties}

We can estimate the statistical uncertainties, on the tSZ-X correlation, using

- our prediction for a tSZ-X cross-correlation power spectrum from the galaxy cluster, $C_{\ell}^{y x}$;

- our prediction for tSZ and X-ray auto-correlation power spectra from the galaxy cluster, $C_{\ell}^{y y}$ and $C_{\ell}^{x x}$;

- the measured cross-correlation, $C_{\ell}^{T_{\nu} \mathrm{R}}$, between the microwave sky from the PSM, denoted $T_{v}$ for frequency $v$, and the X-ray sky from RASS data, denoted R;

- the measured auto correlations, $C_{\ell}^{T_{v} T_{v}}$ and $C_{\ell}^{\mathrm{RR}}$, of the microwave and X-ray skies;

- the measured cross correlation, $C_{\ell}^{y_{\mathrm{PSM}} \mathrm{R}}$, between the tSZ map constructed from the PSM, denoted $y_{\mathrm{PSM}}$, and the X-ray sky from RASS data, denoted R;

- the measured auto correlation, $C_{\ell}^{y_{\mathrm{PSM} y \mathrm{PSM}}}$ of the tSZ map.

The expression uncertainties for each $\mathrm{tSZ}-\mathrm{X}$ detection method is presented in Sects. 4.3, 4.4, and 4.5.

Our estimation of uncertainties through simulations of the microwave sky does not allows an estimate of the systematic uncertainties. To account for systematics uncertainties, we considered three cases for describing the measured tSZ-X cross correlation.

- Case 1: considering only the contribution of galaxy clusters to the tSZ-X correlation.

- Case 2: considering the contributions from galaxy clusters and AGNs to tSZ-X correlation.

- Case 3: considering galaxy clusters, AGNs, and CIB-X contributions.

The complete description of the cross power spectrum for Case 3 reads as

$C_{\ell}=\left[A_{\mathrm{cl}} g(v)+A_{\mathrm{CIB}} F_{\mathrm{CIB}}(v)\right] C_{\ell}^{y x}+A_{\mathrm{AGN}} F_{\mathrm{AGN}}(v)$,

with $F_{\mathrm{CIB}}$ the CIB SED (Gispert et al. 2000), $F_{\mathrm{AGN}}(v)$ a typical radio-loud $A G N$ SED assuming a spectral index of -0.7 in intensity units, $A_{\mathrm{cl}}$ (see Eq. (28)), $A_{\mathrm{CIB}}$, and $A_{\mathrm{AGN}}$ are the parameters of the model and account for galaxy clusters, CIB-X, and AGN contributions to the $\mathrm{tSZ}-\mathrm{X}$ cross power spectrum, respectively. Case 1 assumes $A_{\mathrm{CIB}}=0$ and $A_{\mathrm{AGN}}=0$ and Case 2 assumes $A_{\mathrm{CIB}}=0$.

This modeling assumes that the CIB-X correlation presents a similar shape, as a function of $\ell$, to the clusters' contribution to the tSZ-X cross spectrum and assumes that the AGN contribution is Poissonian, since the AGN clustering can be neglected.

In the following, we consider the multipole range $40<\ell<$ 2000 for our signal-to-noise ratio prediction.

\subsection{Cross correlation spectrum from frequency maps}

The tSZ-X cross correlation can be directly estimated from the correlation between X-ray maps and microwave full-sky observations at a given frequency, denoted $C_{\ell}^{\nu R}$. We estimate the expected level of uncertainties when correlating the RASS hardband and microwave maps at frequency $v$. We mask $30 \%$ of the 

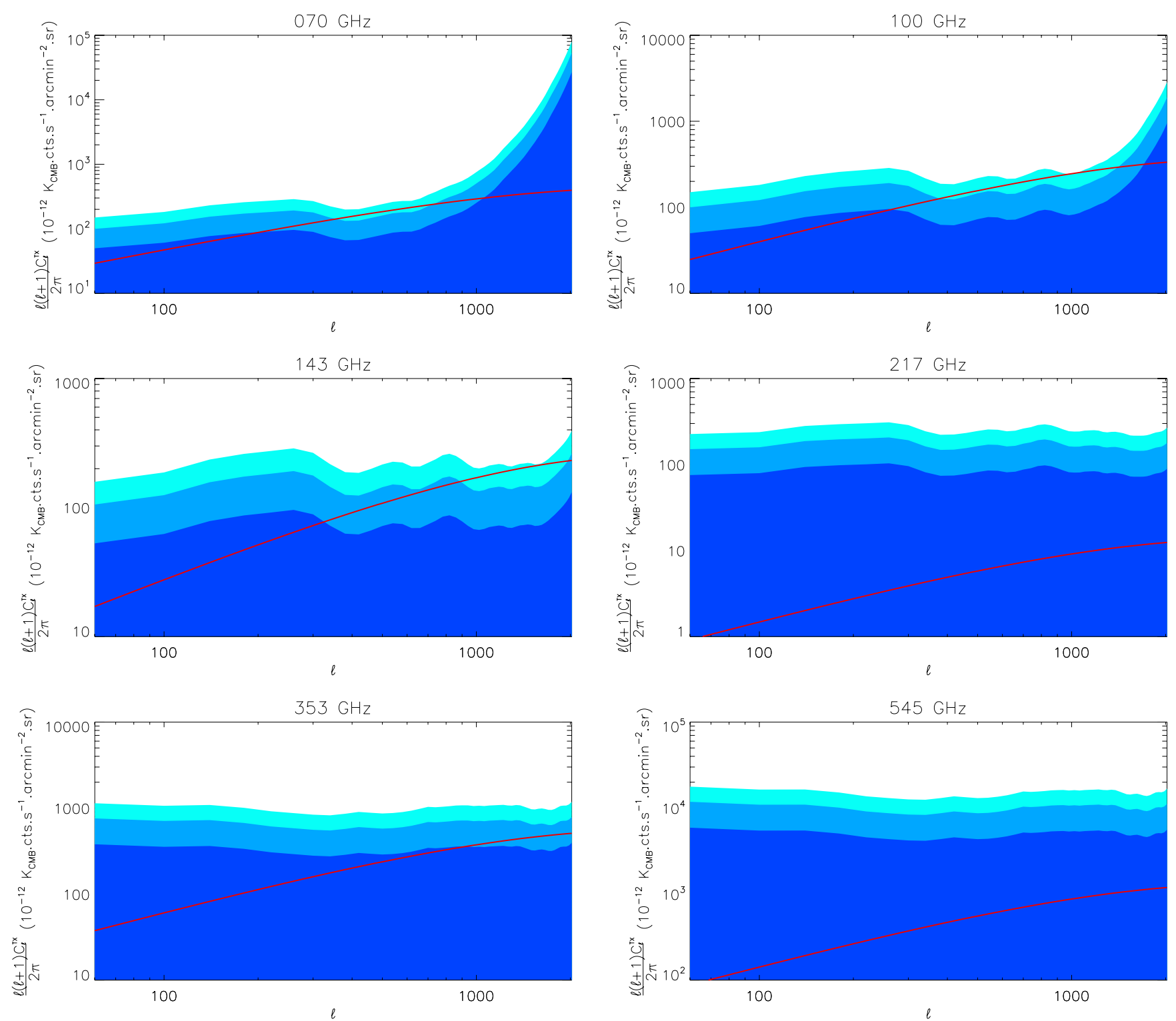

Fig. 7. From left to right and top to bottom: predicted sensitivity to the tSZ-X cross correlation by computing the cross power spectra between the RASS hard band and the Planck channels from 70 to $545 \mathrm{GHz}$. The dark-blue, blue, and light-blue shaded regions show the uncertainties levels at 1,2 , and $3 \sigma$ for multipole bins $\Delta \ell=40$. The absolute value of the theoretical tSZ-X cross angular power spectrum is displayed as a red line.

sky by applying a cut on the thermal dust emission intensity. Then, we estimate the uncertainties following

$$
\begin{aligned}
\left(\Delta C_{\ell}^{v R}\right)^{2}= & \frac{g(v)^{2}\left[\left(C_{\ell}^{y x}\right)^{2}+C_{\ell}^{y y} C_{\ell}^{x x}\right]}{(2 \ell+1) f_{\text {sky }}} \\
& +\frac{\left(C_{\ell}^{T_{\nu} \mathrm{R}}\right)^{2}}{(2 \ell+1) f_{\text {sky }}} \\
& +\frac{\left(C_{\ell}^{T_{\nu} T_{v}}+g(v)^{2} C_{\ell}^{y y}\right)\left(C_{\ell}^{\mathrm{RR}}-C_{\ell}^{x x}\right)}{(2 \ell+1) f_{\text {sky }}},
\end{aligned}
$$

where $\Delta C_{\ell}^{\nu R}$ is the uncertainties on the cross power spectrum between microwave and $\mathrm{X}$-ray skies, and $f_{\text {sky }}$ is the sky fraction used for the analysis. The first term in Eq. (30) corresponds to the cosmic variance of the tSZ-X cross correlation, and the other terms account for the uncertainties produced by foreground emissions.

In Fig. 7, we present the resulting uncertainty level at 1, 2, and $3 \sigma$ as a function of the frequency. We also present the expected absolute value of the tSZ-X cross correlation for our fiducial model. All spectra are displayed in units of $\mathrm{K}_{\mathrm{CMB}}$ cts s$^{-1} \operatorname{arcmin}^{-2}$ sr. Each spectrum has been corrected for the mask effect (see Tristram et al. 2005) and the beam effects. We chose to present the uncertainty for multipole bins with $\Delta \ell=40$.

For the lowest frequencies, below $70 \mathrm{GHz}$, the signal is completely dominated by the instrumental noise contribution. For intermediate frequencies from 70 to $217 \mathrm{GHz}$, the main uncertainty is the CMB contamination, and the uncertainty level clearly shows the CMB features, mainly the first three acoustic pics. Above $353 \mathrm{GHz}$, the uncertainties are dominated by the thermal dust contamination. 
Table 4. Detection signal-to-noise of the tSZ-X cross power spectrum frequency per frequency from 30 to $857 \mathrm{GHz}$ considering values of $\ell$ from 40 to 2000 .

\begin{tabular}{ccccccccc}
\hline \hline Frequency $(\mathrm{GHz})$ & 30 & 44 & 70 & 100 & 143 & 217 & 353 & 545 \\
\hline Case 1 & 3.4 & 4.5 & 8.9 & 13.9 & 15.2 & 0.8 & 7.7 & 1.2 \\
Case 2 & 1.4 & 1.8 & 4.2 & 6.4 & 6.3 & 0.3 & 3.2 & 0.5 \\
\hline
\end{tabular}

We note that at $217 \mathrm{GHz}$ the tSZ emission is not rigorously zero; however, the tSZ transmission in this channel is faint (Hurier et al. 2014). Consequently, this channel can be used to check systematic effects.

In Table 4, we present the expected signal-to-noise for $\mathrm{tSZ}-\mathrm{X}$ correlation as a function of frequency. The signal-to-noise is provided assuming Cases 1 and 2. We observe that in Case 3, we reach a signal-to-noise above six for only two channels, 100 and $143 \mathrm{GHz}$. However, the main limitation in that case is the astrophysical emission from the microwave sky, such as CMB and thermal dust emission. These emissions are correlated from one frequency to another.

\subsection{Cross power spectrum from cleaned frequency maps}

To increase the signal to noise of the tSZ-X detection, we combine the different frequencies to remove the contribution from $\mathrm{CMB}$ and thermal dust. This cleaning is performed by subtracting the $217 \mathrm{GHz}$ spectrum to other spectra to remove CMB contamination and decorrelating each channel from the $857 \mathrm{GHz}$ map to reduce thermal dust contamination.

$\tilde{C}_{\ell}^{T_{\nu} \mathrm{R}}=C_{\ell}^{T_{\nu} \mathrm{R}}-C_{\ell}^{T_{217} \mathrm{R}}-\left(\rho_{v}-\rho_{217}\right) C_{\ell}^{T_{857} \mathrm{R}}$,

where $\rho_{v}$ is the correlation factor between the map at the frequency $v$ and the map at $857 \mathrm{GHz}$. This factor is computed on the area of the sky that is not masked. We computed these cleaned angular power spectra at 70, 100, 143, 353, and $545 \mathrm{GHz}$.

We propagate the uncertainties considering the correlation between cross spectra,

$$
\begin{aligned}
\left\langle C_{\ell}^{\nu \mathrm{R}}, C_{\ell}^{\nu^{\prime} \mathrm{R}}\right\rangle= & \frac{g(v) g\left(v^{\prime}\right)\left[\left(C_{\ell}^{y x}\right)^{2}+C_{\ell}^{y y} C_{\ell}^{x x}\right]}{(2 \ell+1) f_{\text {sky }}} \\
& +\frac{C_{\ell}^{T_{\ell} \mathrm{R}} C_{\ell}^{T_{\ell^{\prime}} \mathrm{R}}}{(2 \ell+1) f_{\text {sky }}} \\
& +\frac{\left(C_{\ell}^{T_{v} T_{\nu^{\prime}}}+g(v) g\left(v^{\prime}\right) C_{\ell}^{y y}\right)\left(C_{\ell}^{\mathrm{RR}}-C_{\ell}^{x x}\right)}{(2 \ell+1) f_{\text {sky }}} .
\end{aligned}
$$

In Fig. 8, we present the obtained power spectrum. We observe that the uncertainties at low $\ell$ are dominated by foreground residuals. Indeed, the decorrelation from the $857 \mathrm{GHz}$ assuming a single scaling coefficient $\rho_{v}$ does not account for thermal dust SED variation across the sky and thus leads to residual emission that dominates the uncertainties on those scales. At high- $\ell$ the uncertainties are dominated by the instrumental noise. In Fig. 8, we also observe that our cleaning is particularly efficient on intermediate scales from $\ell=100$ to $\ell=1000$, owing to CMB contamination removal.

In Table 5 we present the expected signal-to-noise of the tSZ-X cross power spectrum signal in the multipole range $40<$ $\ell<2000$. We provide the results in the three cases described in Sect. 4.2 .

For Case 2, we performed the adjustment of $A_{\mathrm{AGN}}$ individually per frequency. For Case 3, we performed the estimation of the expected signal-to-noise ratio per frequency and considered all frequencies. We note that for both adjustments, the parameters $A_{\mathrm{CIB}}$ and $A_{\mathrm{AGN}}$ are fit considering all frequencies. This adjustment is performed considering the global covariance matrix of all spectra.

This procedure explains the increase in signal-to-noise between Cases 2 and 3. As for Case 3, we consider the multifrequency information of the tSZ-X cross correlation for the fit of our foreground model.

In a realistic case, Case 3, we reach a signal-to-noise of 13.4 for the tSZ-X cross correlation at $143 \mathrm{GHz}$. Considering all five frequencies, we obtain a global signal-to-noise of 17.5.

\subsection{Cross-correlation from Compton parameter maps}

We construct a tSZ map from the PSM simulations of microwave sky observations from 100 to $857 \mathrm{GHz}$ using the MILCA method (Hurier et al. 2013). Then, we estimate the uncertainties on the cross power spectrum, $C_{\ell}^{y \mathrm{R}}$, between a tSZ map and an X-ray map as

$$
\begin{aligned}
& \left(\Delta C_{\ell}^{y \mathrm{R}}\right)^{2}=\frac{\left(C_{\ell}^{y x}\right)^{2}+C_{\ell}^{y y} C_{\ell}^{x x}}{(2 \ell+1) f_{s k y}} \\
& +\frac{\left(C_{\ell}^{y_{\mathrm{PSM}} \mathrm{R}}\right)^{2}+\left(C_{\ell}^{y_{\mathrm{PSM}} y_{\mathrm{PSM}}}+C_{\ell}^{y y}\right)\left(C_{\ell}^{\mathrm{RR}}-C_{\ell}^{x x}\right)}{(2 \ell+1) f_{s k y}},
\end{aligned}
$$

where $y_{\mathrm{PSM}}$ corresponds to the tSZ map constructed from the PSM simulations.

In Fig. 9, we present the obtained levels of uncertainties. The tSZ-X power spectrum for our fiducial model is above $2 \sigma$ for each bin of $\Delta \ell=40$ from $\ell=40$ to 2000. The main limitations are the instrumental noise and CIB residuals that cannot be removed by a linear combination. If we do not consider contamination by correlated astrophysical emissions such as radioloud AGNs and CIB, we obtain an overall signal-to-noise of 62.3 from $\ell=40$ to $\ell=2000$. If we consider contamination by AGNs and CIB we obtain a signal-to-noise of 31.5.

We note in Sect. 3.5 that the modeling derived from present constraints leads to about a $48 \%$ uncertainty on the amplitude of the tSZ-X cross correlation. Considering Case 3 and a signal-tonoise of 31.5 , the amplitude of the tSZ-X cross-correlation can be obtained at $3.2 \%$ precision. As a consequence, the utilization of a tSZ map allows to set the tighter constraints on the tSZ-X cross-correlation and to increase our knowledge of cosmological and astrophysical parameters in the related degeneracy space by a factor of 15 .

\subsection{Constraints on astrophysical and cosmological parameters}

The amplitude of the tSZ-X cross correlation can be constraints at a precision of $3.2 \%$. However, this amplitude is sensitive to several parameters from both cosmology and scaling laws, see 

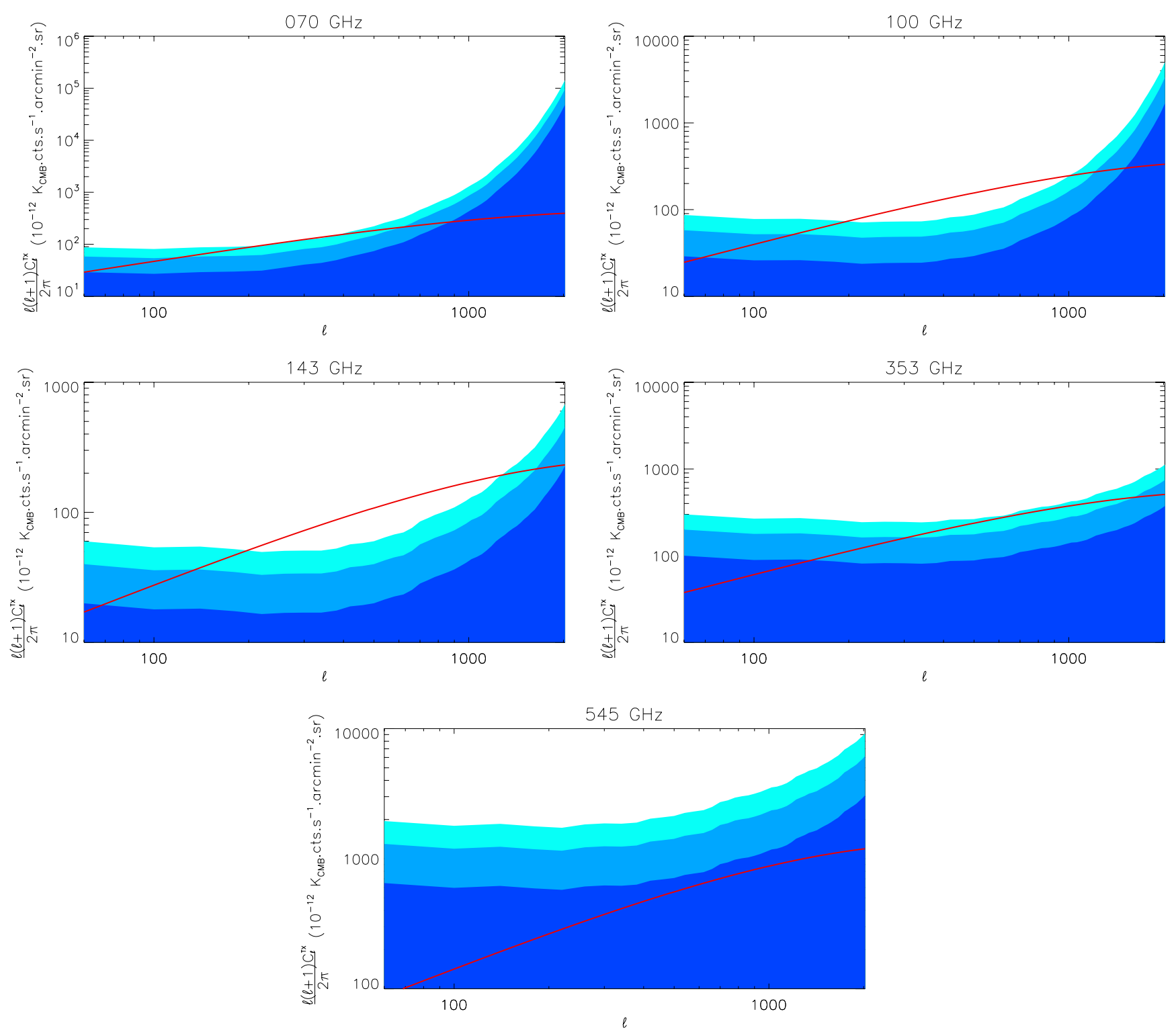

Fig. 8. From left to right and top to bottom: predicted sensitivity to the tSZ-X cross correlation for the cleaned cross power spectra between the RASS hard band and the Planck channels at 70, 100, 143, 353, and $545 \mathrm{GHz}$, respectively. The dark-blue, blue, and light-blue shaded regions show the uncertainty levels at 1,2 , and $3 \sigma$ for multipole bins $\Delta \ell=40$. The absolute value of the theoretical tSZ-X cross angular power spectrum is displayed as a red line.

Table 5. Detection signal-to-noise of the tSZ-X cross power spectrum frequency per frequency after cleaning at 70, 100, 143, 353, and 545 GHz considering values of $\ell$ from 40 to 2000 .

\begin{tabular}{ccccccc}
\hline \hline Frequency $(\mathrm{GHz})$ & 70 & 100 & 143 & 353 & 545 & All \\
\hline Case 1 & 8.5 & 16.3 & 19.8 & 13.5 & 3.9 & - \\
Case 2 & 3.9 & 7.1 & 8.9 & 6.0 & 1.8 & - \\
Case 3 & 4.7 & 9.4 & 13.4 & 11.8 & 3.0 & 17.5 \\
\hline
\end{tabular}

Eq. (28). The expected constraints on the tSZ-X cross correlation normalization reads

$\Delta A_{\mathrm{cl}}=0.03$,

in the case of the correlation between a tSZ map and an Xray map considering the contamination by both AGN and CIB emissions.

To obtain the uncertainty over a single parameter, it is necessary for it to carefully propagate the uncertainties from other parameters considering the global covariance matrix of all parameters. Beyond the amplitude of the tSZ-X cross-correlation, if we fix the profiles of galaxy clusters, the expected high level of significance of the tSZ-X cross correlation should allow us to constrain the scaling-law spectral indices.

In Fig. 10, we present the constraints from our simulated data. The tSZ-X cross correlation is only sensitive to the sum $\delta \alpha_{\mathrm{x}}+\delta \alpha_{\mathrm{sz}}$ for fixed density and temperature 


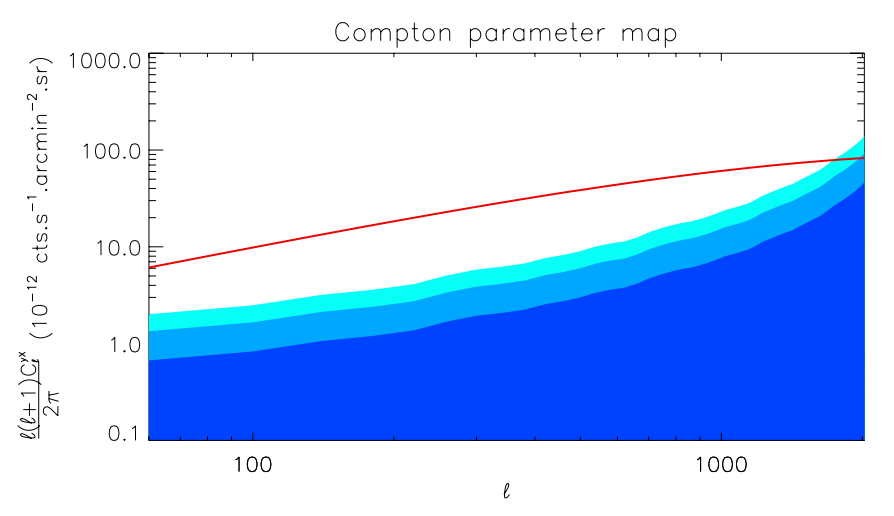

Fig. 9. Predicted sensitivity to the tSZ-X cross-correlation for the cross power spectra between the RASS hard band and a MILCA tSZ-map. The dark-blue, blue, and light-blue shaded regions show the uncertainties levels at 1,2 , and $3 \sigma$ for multipole bins $\Delta \ell=40$. The absolute value of the theoretical tSZ-X cross angular power spectrum is displayed as a red line.

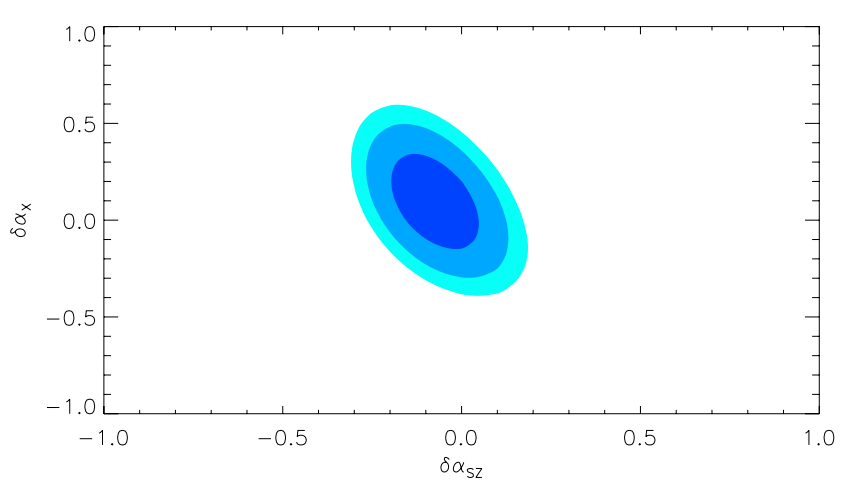

Fig. 10. Likelihood function as a function of the deviation from scaling law indices $\delta \alpha_{\mathrm{sz}}$ and $\delta \alpha_{\mathrm{x}}$ derived from tSZ auto-correlation power spectrum and $\mathrm{tSZ}-\mathrm{X}$ cross power spectrum.

profiles. To distinguish the two indices, we used the tSZ autocorrelation power spectrum assuming uncertainty levels from Planck Collaboration VIII (2011).

This measurement is limited by the multipole range accessible, for example, by Planck and ROSAT, $\ell \leq 2000$. In that case, we obtained $\Delta\left(\delta \alpha_{\mathrm{sz}}\right)=0.10$ and $\Delta\left(\delta \alpha_{\mathrm{sz}}\right)=0.16$ for the scaling law index deviation parameters. If we add a measurement of tSZ spectrum at $\ell=3000$, considering tSZ data at higher resolution from SPT-like (Shirokoff et al. 2011) or ACT-like (Sievers et al. 2013) experiments, we can reach $\Delta\left(\delta \alpha_{\mathrm{sz}}\right)=0.08$ and $\Delta\left(\delta \alpha_{\mathrm{x}}\right)=0.14$. These constraints on the scaling-law slopes with respect to the cluster mass are competitive with constraints from the scaling-law-dedicated analyses presented in Table 1.

Finally, we estimated the degeneracy between profile and scaling-relation parameters considering the tSZ-X cross power spectrum. For small variations (up to 10\%) around the Arnaud et al. (2010) profile, we can express the degeneracy relation as

$\delta\left(\alpha_{\mathrm{sz}}+\alpha_{\mathrm{x}}\right)_{\mathrm{syst}}=0.81 \delta \alpha-0.43 \delta \beta-0.90 \delta \gamma-1.19 \delta c_{500}+0.48 \delta \epsilon_{p}$.

From this result, we observed that the shape of the tSZ-X cross spectrum is highly sensitive to both scaling-relation and profile parameters. Consequently, the $\mathrm{tSZ}-\mathrm{X}$ spectrum is a powerful probe to simultaneously test the combination of scaling laws and profiles.

\section{Conclusion}

We have presented a complete up-to-date modeling of the tSZ, $\mathrm{X}$-ray, and tSZ-X power spectra. We have carefully studied the sensitivity to cosmological and astrophysical parameters, completing a previous analysis on the topic (Diego et al. 2003). For the variations in the tSZ-X cross-correlation, tSZ, and X-ray auto-correlation with $H_{0}, \Omega_{\mathrm{m}}$, and $\sigma_{8}$, we provided accurate analytical fitting formulae in the range $0<\ell<10000$.

Then, we carefully propagated the uncertainties on the cosmological and scaling law parameters of our modeling to the predicted power spectra, leading to an overall uncertainty on the normalization of the tSZ-X power spectrum by about $48 \%$. This result highlights our limited knowledge of this cosmological probes.

We note that the contributions to the total uncertainty from clusters' scaling relations and cosmological parameters are at same order of magnitude. The main contributions to the total uncertainty are produced by the bias $b, 35 \%$, and cosmological parameters, $27 \%$. These large uncertainties illustrate the importance of an accurate measurement of the tSZ-X cross angular spectrum to set constraints on both cluster properties and cosmological parameters.

We stress that most of our modeling parameters act on the tSZ-X power spectrum as an overall amplitude factor. This leads to high degeneracy between those parameters, and thus the tSZ-X cross correlation needs to be used in addition of other probes to break the degeneracies. We note that our prediction cannot be directly compared to the measurement recently performed by Hajian et al. (2013), since their measurement concerned the cross-correlation between tSZ maps and X-ray cata$\operatorname{logs}$, and consequently, it presents difference dependencies with cosmological parameters.

We predicted the expected signal-to-noise that can be reached using a simulated microwave sky and ROSAT data for the X-ray sky. We considered three approaches to extracting the tSZ-X cross power spectrum. We demonstrate that in the case of the cross-correlation between a tSZ map and an X-ray map, we reach a signal-to-noise of 31.5. In this case, we can reach a measurement of the tSZ-X cross correlation amplitude at about $3 \%$, improving the actual constraints (from our knowledge of cosmological parameters and scaling laws) on the predicted spectrum by about a factor of 10 .

We also studied the possibility of constraining the slope of the tSZ and X-ray scaling laws and the parameters of a GNFW profile using the shape of the tSZ-X cross spectrum. We conclude that scaling-law and profile parameters are highly degenerated. Thus, constraints from tSZ-X spectrum allows the global consistency of scaling laws and profiles to be checked.

Future experiment at high resolution and high sensitivity for tSZ surveys and X-ray measurements will, in the near future, allow the increase of the expected constraints, especially by providing a wider range of multipoles, higher sensitivity, and more frequencies.

Acknowledgements. We acknowledge the support of the French Agence Nationale de la Recherche under grant ANR-11-BD56-015. We are grateful to an anonymous referee for his/her comments and suggestions. We also thank M. Arnaud, J. B. Melin, E. Pointecouteau, and G. Pratt for useful discussions related to this work. This research has made use of the ROSAT all-sky survey data which have been processed at MPE. We acknowledge the use of HEALPix package (Górski et al. 2005). 


\section{References}

Andreon, S. 2012, A\&A, 546, A6

Arnaud, M., Pratt, G. W., Piffaretti, R., et al. 2010, A\&A, 517, A92

Benson, B. A., de Haan, T., Dudley, J. P., et al. 2013, ApJ, 763, 147

Bohringer, H., Voges, W., Huchra, J. P., et al. 2000, VizieR Online Data Catalog, J/MNRAS/318/333

Böhringer, H., Schuecker, P., Pratt, G. W., et al. 2007, A\&A, 469, 363

Delabrouille, J., Betoule, M., Melin, J.-B., et al. 2013, A\&A, 553, A96

Diego, J. M., Silk, J., \& Sliwa, W. 2003, MNRAS, 346, 940

Ebeling, H., Edge, A. C., Allen, S. W., et al. 2000, VizieR Online Data Catalog, J/ApJS/129/435

Ebeling, H., Edge, A. C., \& Henry, J. P. 2001, ApJ, 553, 668

Evrard, A. E., MacFarland, T. J., Couchman, H. M. P., et al. 2002, ApJ, 573, 7

Fixsen, D. J., Dwek, E., Mather, J. C., Bennett, C. L., \& Shafer, R. A. 1998, ApJ, 508,123

Freyberg, M. J., Snowden, S. L., Plucinsky, P. P., \& Schmitt, J. H. M. M. 1992, in Astronomische Gesellschaft Abstract Series, 7, ed. G. Klare, 26

Gispert, R., Lagache, G., \& Puget, J. L. 2000, A\&A, 360, 1

Górski, K. M., Hivon, E., Banday, A. J., et al. 2005, ApJ, 622, 759

Hajian, A., Battaglia, N., Spergel, D. N., et al. JCAP, 11, 064

Hurier, G., Macías-Pérez, J. F., \& Hildebrandt, S. 2013, A\&A, 558, A118

Hurier, G., Aghanim, N., Douspis, M., \& Pointecouteau, E. 2014, A\&A, 561, A143

Komatsu, E., \& Kitayama, T. 1999, ApJ, 526, L1

Komatsu, E., \& Seljak, U. 2001, MNRAS, 327, 1353

Komatsu, E., \& Seljak, U. 2002, MNRAS, 336, 1256

Krumpe, M., Miyaji, T., \& Coil, A. L. 2010, ApJ, 713, 558

Krumpe, M., Miyaji, T., Coil, A. L., \& Aceves, H. 2012, ApJ, 746, 1

Lesgourgues, J. 2011, unpublished [arXiv: 1104 . 2932]

Marriage, T. A., Acquaviva, V., Ade, P. A. R., et al. 2011, ApJ, 737, 61

Mewe, R., Gronenschild, E. H. B. M., \& van den Oord, G. H. J. 1985, A\&AS,

62, 197

Miyaji, T., Krumpe, M., Coil, A. L., \& Aceves, H. 2011, ApJ, 726, 83
Mo, H. J., \& White, S. D. M. 1996, MNRAS, 282, 347

Nagai, D., Kravtsov, A. V., \& Vikhlinin, A. 2007, ApJ, 668, 1

Navarro, J. F., Frenk, C. S., \& White, S. D. M. 1997, ApJ, 490, 493

Piffaretti, R., \& Valdarnini, R. 2008, A\&A, 491, 71

Planck Collaboration VIII. 2011, A\&A, 536, A8

Planck Collaboration XI. 2011, A\&A, 536, A11

Planck Collaboration Int. V. 2013, A\&A, 550, A131

Planck Collaboration I. 2014, A\&A, in press, DOI: 10.1051/0004-6361/201321529

Planck Collaboration XVI. 2014, A\&A, in press, DOI: $10.1051 / 0004-6361 / 201321591$

Planck Collaboration XX. 2014, A\&A, in press, DOI: 10.1051/0004-6361/201321521

Planck Collaboration XXIX. 2014, A\&A, in press, DOI: 10.1051/0004-6361/201321523

Puget, J.-L., Abergel, A., Bernard, J.-P., et al. 1996, A\&A, 308, L5

Reichardt, C. L., Stalder, B., Bleem, L. E., et al. 2013, ApJ, 763, 127

Reichert, A., Böhringer, H., Fassbender, R., \& Mühlegger, M. 2011, A\&A, 535, A4

Remazeilles, M., Delabrouille, J., \& Cardoso, J.-F. 2011, MNRAS, 410, 2481

Sayers, J., Czakon, N. G., Mantz, A., et al. 2013, ApJ, 768, 177

Sehgal, N., Trac, H., Acquaviva, V., et al. 2011, ApJ, 732, 44

Shirokoff, E., Reichardt, C. L., Shaw, L., et al. 2011, ApJ, 736, 61

Sievers, J. L., Hlozek, R. A., Nolta, M. R., et al. 2013, Cosmol. Astropart. Phys., 10,60

Sunyaev, R. A., \& Zeldovich, Y. B. 1969, Nature, 223, 721

Sunyaev, R. A., \& Zeldovich, Y. B. 1972, Comments Astrophys. Space Phys., 4, 173

Taburet, N., Hernández-Monteagudo, C., Aghanim, N., Douspis, M., \& Sunyaev, R. A. 2011, MNRAS, 418, 2207

Tinker, J., Kravtsov, A. V., Klypin, A., et al. 2008, ApJ, 688, 709

Tristram, M., Macías-Pérez, J. F., Renault, C., \& Santos, D. 2005, MNRAS, 358, 833

Vanderlinde, K., Crawford, T. M., de Haan, T., et al. 2010, ApJ, 722, 1180

Voges, W., Aschenbach, B., Boller, T., et al. 1999, A\&A, 349, 389 